\title{
A precision measurement of the inclusive $e p$ scattering cross section at HERA
}

The H1 Collaboration

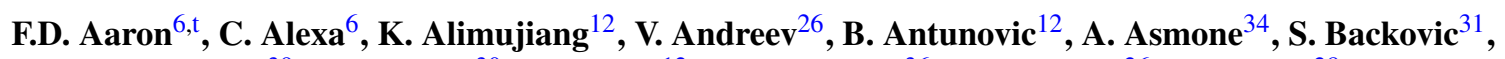
A. Baghdasaryan ${ }^{39}$, E. Barrelet ${ }^{30}$, W. Bartel ${ }^{12}$, K. Begzsuren ${ }^{36}$, A. Belousov ${ }^{26}$, J.C. Bizot $^{28}$, V. Boudry ${ }^{29}$, I. Bozovic-Jelisavcic ${ }^{2}$, J. Bracinik ${ }^{3}$, G. Brandt ${ }^{12}$, M. Brinkmann ${ }^{13}$, V. Brisson ${ }^{28}$, D. Bruncko ${ }^{17}$, A. Bunyatyan ${ }^{14,39}$, G. Buschhorn $^{27}$, L. Bystritskaya ${ }^{25}$, A.J. Campbell ${ }^{12}$, K.B. Cantun Avila ${ }^{23}$, F. Cassol-Brunner ${ }^{22}$, K. Cerny ${ }^{33}$, V. Cerny ${ }^{17, \mathrm{r}}$, V. Chekelian ${ }^{27}$, A. Cholewa ${ }^{12}$, J.G. Contreras $^{23}$, J.A. Coughlan ${ }^{7}$, G. Cozzika ${ }^{11}$, J. Cvach ${ }^{32}$, J.B. Dainton ${ }^{19}$, K. Daum ${ }^{38, n}$, M. Deák ${ }^{12}$, Y. de Boer ${ }^{12}$, B. Delcourt ${ }^{28}$, M. Del Degan ${ }^{41}$, J. Delvax ${ }^{4,5}$, A. De Roeck ${ }^{12, p}$, E.A. De Wolf ${ }^{4,5}$, C. Diaconu ${ }^{22}$, V. Dodonov ${ }^{14}$, A. Dossanov ${ }^{27}$, A. Dubak ${ }^{31, q}$, G. Eckerlin ${ }^{12}$, V. Efremenko ${ }^{25}$, S. Egli ${ }^{37}$, A. Eliseev ${ }^{26}$, E. Elsen ${ }^{12}$, A. Falkiewicz ${ }^{8}$, P.J.W. Faulkner ${ }^{3}$, L. Favart ${ }^{4,5}$, A. Fedotov ${ }^{25}$, R. Felst ${ }^{12}$, J. Feltesse ${ }^{11, \mathrm{~s}}$, J. Ferencei ${ }^{17}$, D.-J. Fischer ${ }^{12}$, M. Fleischer ${ }^{12}$, A. Fomenko ${ }^{26}$, E. Gabathuler ${ }^{19}$, J. Gayler ${ }^{12}$, S. Ghazaryan ${ }^{39}$, A. Glazov ${ }^{12}$, I. Glushkov ${ }^{40}$, L. Goerlich ${ }^{8}$, N. Gogitidze ${ }^{26}$, M. Gouzevitch ${ }^{12}$, C. Grab ${ }^{41}$, T. Greenshaw ${ }^{19}$, B.R. Grell ${ }^{12}$, G. Grindhammer $^{27}$, S. Habib ${ }^{13, \text { u }}$, D. Haidt ${ }^{12}$, C. Helebrant ${ }^{12}$, R.C.W. Henderson ${ }^{18}$, E. Hennekemper ${ }^{16}$, H. Henschel ${ }^{40}$, M. Herbst ${ }^{16}$, G. Herrera ${ }^{24}$, M. Hildebrandt ${ }^{37}$, K.H. Hiller ${ }^{40}$, D. Hoffmann ${ }^{22}$, R. Horisberger ${ }^{37}$, T. Hreus ${ }^{4,5,0}$, M. Jacquet ${ }^{28}$, M.E. Janssen ${ }^{12}$, X. Janssen ${ }^{4,5}$, V. Jemanov ${ }^{13}$, L. Jönsson ${ }^{21}$, A.W. Jung ${ }^{16}$, H. Jung ${ }^{12}$, M. Kapichine ${ }^{10}$, J. Katzy ${ }^{12}$, I.R. Kenyon ${ }^{3}$, C. Kiesling ${ }^{27}$, M. Klein ${ }^{19}$, C. Kleinwort ${ }^{12}$, T. Kluge ${ }^{19}$, A. Knutsson ${ }^{12}$, R. Kogler ${ }^{27}$, V. Korbel ${ }^{12}$, P. Kostka $^{40}$, M. Kraemer ${ }^{12}$, K. Krastev ${ }^{12}$, J. Kretzschmar ${ }^{19}$, A. Kropivnitskaya ${ }^{25}$,

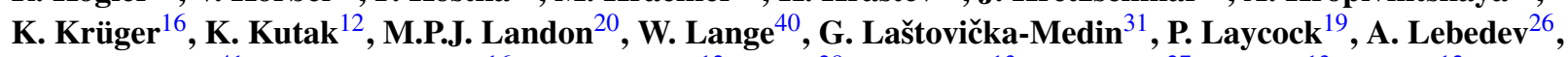

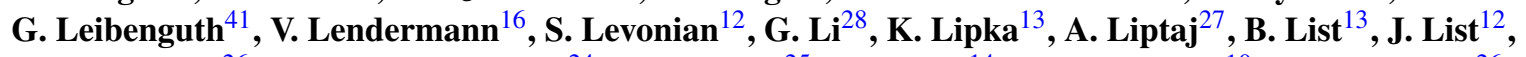
N. Loktionova ${ }^{26}$, R. Lopez-Fernandez ${ }^{24}$, V. Lubimov ${ }^{25}$, L. Lytkin $^{14}$, A. Makankine ${ }^{10}$, E. Malinovski ${ }^{26}$, P. Marage ${ }^{4,5}$, Ll. Marti ${ }^{12}$, H.-U. Martyn ${ }^{1}$, S.J. Maxfield ${ }^{19}$, A. Mehta $^{19}$, A.B. Meyer ${ }^{12}$, H. Meyer ${ }^{12}$, H. Meyer ${ }^{38}$, J. Meyer ${ }^{12}$, V. Michels ${ }^{12}$, S. Mikocki ${ }^{8}$, I. Milcewicz-Mika ${ }^{8}$, F. Moreau $^{29}$, A. Morozov ${ }^{10}$, J.V. Morris ${ }^{7}$, M.U. Mozer ${ }^{4,5}$, M. Mudrinic ${ }^{2}$, K. Müller ${ }^{42}$, P. Murín ${ }^{17,0}$, B. Naroska ${ }^{13, \mathrm{x}}$, Th. Naumann ${ }^{40}$, P.R. Newman ${ }^{3}$, C. Niebuhr ${ }^{12}$, A. Nikiforov ${ }^{12}$, G. Nowak $^{8}$, K. Nowak ${ }^{42}$, M. Nozicka' ${ }^{12}$, B. Olivier ${ }^{27}$, J.E. Olsson ${ }^{12}$, S. Osman ${ }^{21}$, D. Ozerov ${ }^{25}$, V. Palichik ${ }^{10}$, I. Panagoulias ${ }^{12,1, \mathrm{~m}}$, M. Pandurovic ${ }^{2}$, Th. Papadopoulou ${ }^{12,1, \mathrm{~m}}$, C. Pascaud $^{28}$, G.D. Patel ${ }^{19}$, O. Pejchal ${ }^{33}$, E. Perez ${ }^{11, p}$, A. Petrukhin ${ }^{25}$, I. Picuric ${ }^{31}$, S. Piec ${ }^{40}$, D. Pitzl ${ }^{12}$, R. Plačakyte ${ }^{12}$, B. Pokorny ${ }^{13}$, R. Polifka ${ }^{33}$, B. Povh ${ }^{14}$, T. Preda ${ }^{6}$, V. Radescu ${ }^{12}$, A.J. Rahmat ${ }^{19}$, N. Raicevic ${ }^{31}$, A. Raspiareza ${ }^{27}$, T. Ravdandorj ${ }^{36}$, P. Reimer $^{32}$, E. Rizvi ${ }^{20}$, P. Robmann ${ }^{42}$, B. Roland ${ }^{4,5}$, R. Roosen ${ }^{4,5}$, A. Rostovtsev $^{25}$, M. Rotaru ${ }^{6}$, J.E. Ruiz Tabasco ${ }^{23}$, Z. Rurikova ${ }^{12}$, S. Rusakov $^{26}$, D. Š́lek ${ }^{33}$, D.P.C. Sankey ${ }^{7}$, M. Sauter ${ }^{41}$, E. Sauvan ${ }^{22}$, S. Schmitt $^{12, y}$, C. Schmitz ${ }^{42}$, L. Schoeffel ${ }^{11}$, A. Schöning ${ }^{15}$, H.-C. Schultz-Coulon ${ }^{16}$, F. Sefkow ${ }^{12}$, R.N. Shaw-West ${ }^{3}$, I. Sheviakov ${ }^{26}$, L.N. Shtarkov ${ }^{26}$, S. Shushkevich ${ }^{27}$, T. Sloan $^{18}$, I. Smiljanic ${ }^{2}$, Y. Soloviev ${ }^{26}$, P. Sopicki ${ }^{8}$, D. South ${ }^{9}$, V. Spaskov ${ }^{10}$, A. Specka ${ }^{29}$, Z. Staykova ${ }^{12}$, M. Steder ${ }^{12}$, B. Stella ${ }^{34}$, G. Stoicea ${ }^{6}$, U. Straumann ${ }^{42}$, D. Sunar ${ }^{4,5}$, T. Sykora ${ }^{4,5}$, V. Tchoulakov ${ }^{10}$,

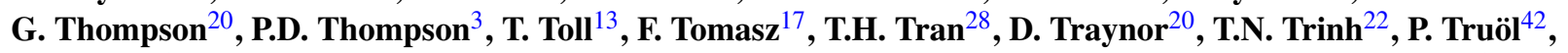
I. Tsakov ${ }^{35}$, B. Tseepeldorj ${ }^{36, v}$, J. Turnau ${ }^{8}$, K. Urban ${ }^{16}$, A. Valkárová ${ }^{33}$, C. Vallée ${ }^{22}$, P. Van Mechelen ${ }^{4,5}$, A. Vargas Trevino $^{12}$, Y. Vazdik ${ }^{26}$, S. Vinokurova ${ }^{12}$, V. Volchinski ${ }^{39}$, M. von den Driesch $^{12}$, D. Wegener ${ }^{9}$, R. Wallny ${ }^{42, w}$, Ch. Wissing ${ }^{12}$, E. Wünsch ${ }^{12}$, J. Žáček ${ }^{33}$, J. Zálešák ${ }^{32}$, Z. Zhang ${ }^{28}$, A. Zhokin ${ }^{25}$, T. Zimmermann ${ }^{41}$, H. Zohrabyan ${ }^{39}$, F. Zomer ${ }^{28}$, R. Zus ${ }^{6}$

\footnotetext{
${ }^{1}$ I. Physikalisches Institut der RWTH, Aachen, Germany ${ }^{\mathrm{a}}$

${ }^{2}$ Vinca Institute of Nuclear Sciences, Belgrade, Serbia

${ }^{3}$ School of Physics and Astronomy, University of Birmingham, Birmingham, $\mathrm{UK}^{\mathrm{b}}$

${ }^{4}$ Inter-University Institute for High Energies ULB-VUB, Brussels, Belgium ${ }^{\mathrm{c}}$

${ }^{5}$ Universiteit Antwerpen, Antwerpen, Belgium ${ }^{\mathrm{c}}$

${ }^{6}$ National Institute for Physics and Nuclear Engineering (NIPNE), Bucharest, Romania

${ }^{7}$ Rutherford Appleton Laboratory, Chilton, Didcot, UK ${ }^{\mathrm{b}}$

${ }^{8}$ Institute for Nuclear Physics, Cracow, Poland ${ }^{\mathrm{d}}$

${ }^{9}$ Institut für Physik, TU Dortmund, Dortmund, Germany ${ }^{\mathrm{a}}$
} 
${ }^{10}$ Joint Institute for Nuclear Research, Dubna, Russia

${ }^{11} \mathrm{CEA}$, DSM/Irfu, CE-Saclay, Gif-sur-Yvette, France

${ }^{12}$ DESY, Hamburg, Germany

${ }^{13}$ Institut für Experimentalphysik, Universität Hamburg, Hamburg, Germany ${ }^{a}$

${ }^{14}$ Max-Planck-Institut für Kernphysik, Heidelberg, Germany

${ }^{15}$ Physikalisches Institut, Universität Heidelberg, Heidelberg, Germany ${ }^{a}$

${ }^{16}$ Kirchhoff-Institut für Physik, Universität Heidelberg, Heidelberg, Germany ${ }^{\mathrm{a}}$

${ }^{17}$ Institute of Experimental Physics, Slovak Academy of Sciences, Košice, Slovak Republic ${ }^{\mathrm{f}}$

${ }^{18}$ Department of Physics, University of Lancaster, Lancaster, UK ${ }^{\mathrm{b}}$

${ }^{19}$ Department of Physics, University of Liverpool, Liverpool, $\mathrm{UK}^{\mathrm{b}}$

${ }^{20}$ Queen Mary and Westfield College, London, UK ${ }^{\mathrm{b}}$

${ }^{21}$ Physics Department, University of Lund, Lund, Sweden ${ }^{\mathrm{g}}$

${ }^{22}$ CPPM, CNRS/IN2P3, Univ. Mediterranee, Marseille, France

${ }^{23}$ Departamento de Fisica Aplicada, CINVESTAV, Mérida, Yucatán, México ${ }^{j}$

${ }^{24}$ Departamento de Fisica, CINVESTAV, México, México ${ }^{j}$

${ }^{25}$ Institute for Theoretical and Experimental Physics, Moscow, Russia ${ }^{\mathrm{k}}$

${ }^{26}$ Lebedev Physical Institute, Moscow, Russia ${ }^{\mathrm{e}}$

${ }^{27}$ Max-Planck-Institut für Physik, München, Germany

${ }^{28}$ LAL, Univ. Paris-Sud, CNRS/IN2P3, Orsay, France

${ }^{29}$ LLR, Ecole Polytechnique, IN2P3-CNRS, Palaiseau, France

${ }^{30}$ LPNHE, Universités Paris VI and VII, IN2P3-CNRS, Paris, France

${ }^{31}$ Faculty of Science, University of Montenegro, Podgorica, Montenegro ${ }^{\mathrm{e}}$

${ }^{32}$ Institute of Physics, Academy of Sciences of the Czech Republic, Praha, Czech Republic ${ }^{\mathrm{h}}$

${ }^{33}$ Faculty of Mathematics and Physics, Charles University, Praha, Czech Republic ${ }^{\text {h }}$

${ }^{34}$ Dipartimento di Fisica Università di Roma Tre and INFN Roma 3, Roma, Italy

${ }^{35}$ Institute for Nuclear Research and Nuclear Energy, Sofia, Bulgaria ${ }^{\mathrm{e}}$

${ }^{36}$ Institute of Physics and Technology of the Mongolian Academy of Sciences, Ulaanbaatar, Mongolia

${ }^{37}$ Paul Scherrer Institut, Villigen, Switzerland

${ }^{38}$ Fachbereich C, Universität Wuppertal, Wuppertal, Germany

${ }^{39}$ Yerevan Physics Institute, Yerevan, Armenia

${ }^{40}$ DESY, Zeuthen, Germany

${ }^{41}$ Institut für Teilchenphysik, ETH, Zürich, Switzerland

${ }^{42}$ Physik-Institut der Universität Zürich, Zürich, Switzerland ${ }^{\mathrm{i}}$

Received: 23 April 2009 / Revised: 21 September 2009 / Published online: 31 October 2009

(C) Springer-Verlag / Società Italiana di Fisica 2009

${ }^{\text {a }}$ Supported by the Bundesministerium für Bildung und Forschung, FRG, under contract numbers $05 \mathrm{H} 1$ 1GUA /1, 05 H1 1PAA /1, 05 H1 1PAB /9, 05 H1 1PEA /6, 05 H1 1VHA /7 and 05 H1 1VHB /5.

${ }^{\mathrm{b}}$ Supported by the UK Science and Technology Facilities Council, and formerly by the UK Particle Physics and Astronomy Research Council. ${ }^{\mathrm{c}}$ Supported by FNRS-FWO-Vlaanderen, IISN-IIKW and IWT and by Interuniversity Attraction Poles Programme, Belgian Science Policy.

${ }^{\mathrm{d}}$ Partially Supported by Polish Ministry of Science and Higher Education, grant PBS/DESY/70/2006.

${ }^{\mathrm{e}}$ Supported by the Deutsche Forschungsgemeinschaft.

${ }^{\text {f }}$ Supported by VEGA SR grant no. 2/7062/ 27.

${ }^{g}$ Supported by the Swedish Natural Science Research Council.

${ }^{\mathrm{h}}$ Supported by the Ministry of Education of the Czech Republic under the projects LC527, INGO-1P05LA259 and MSM0021620859.

${ }^{i}$ Supported by the Swiss National Science Foundation.

${ }^{\mathrm{j} S u p p o r t e d ~ b y ~ C O N A C Y T, ~ M e ́ x i c o, ~ g r a n t ~ 48778-F . ~}$

${ }^{k}$ Russian Foundation for Basic Research (RFBR), grant no 1329.2008.2.

${ }^{1}$ This project is co-funded by the European Social Fund (75\%) and National Resources (25\%)-(EPEAEK II)-PYTHAGORAS II.
${ }^{\mathrm{m}}$ Also at Physics Department, National Technical University, Zografou Campus, GR-15773 Athens, Greece.

${ }^{\mathrm{n}}$ Also at Rechenzentrum, Universität Wuppertal, Wuppertal, Germany.

${ }^{\circ}$ Also at University of P.J. Šafárik, Košice, Slovak Republic.

pAlso at CERN, Geneva, Switzerland.

${ }^{\mathrm{q}}$ Also at Max-Planck-Institut für Physik, München, Germany.

${ }^{\mathrm{r}}$ Also at Comenius University, Bratislava, Slovak Republic.

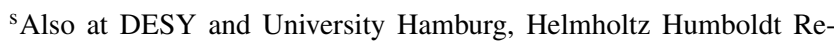
search Award.

${ }^{t}$ Also at Faculty of Physics, University of Bucharest, Bucharest, Romania.

uSupported by a scholarship of the World Laboratory Björn Wiik Research Project.

${ }^{v}$ Also at Ulaanbaatar University, Ulaanbaatar, Mongolia.

${ }^{\mathrm{w}}$ Now at University of California, Los Angeles, United States of America.

${ }^{\mathrm{x}}$ Deceased.

y e-mail: sschmitt@mail.desy.de 
Abstract A measurement of the inclusive deep inelastic neutral current $e^{+} p$ scattering cross section is reported in the region of four-momentum transfer squared, $12 \mathrm{GeV}^{2} \leq$ $Q^{2} \leq 150 \mathrm{GeV}^{2}$, and Bjorken $x, 2 \times 10^{-4} \leq x \leq 0.1$. The results are based on data collected by the H1 Collaboration at the $e p$ collider HERA at positron and proton beam energies of $E_{e}=27.6 \mathrm{GeV}$ and $E_{p}=920 \mathrm{GeV}$, respectively. The data are combined with previously published data, taken at $E_{p}=820 \mathrm{GeV}$. The accuracy of the combined measurement is typically in the range of $1.3-2 \%$. A QCD analysis at next-to-leading order is performed to determine the parton distributions in the proton based on $\mathrm{H} 1$ data.

\section{Introduction}

The electron-proton collider HERA extends the kinematic range of deep inelastic lepton-nucleon scattering (DIS), determined by the four-momentum transfer squared, $Q^{2}$, and Bjorken $x$, by two orders of magnitude towards high $Q^{2}$ and small $x$, compared with fixed target experiments. This allows proton structure to be thoroughly investigated as is vital for the understanding of strong interactions, described by Quantum Chromodynamics (QCD).

This paper presents the most accurate cross section data to date for the inclusive neutral current process $e^{+} p \rightarrow$ $e^{+} X$, measured in the kinematic region $12 \mathrm{GeV}^{2} \leq Q^{2} \leq$ $150 \mathrm{GeV}^{2}$ and $2 \times 10^{-4} \leq x \leq 0.1$. The data newly presented here were taken in the year 2000 with positrons of energy $E_{e}=27.6 \mathrm{GeV}$ and protons of energy $E_{p}=920 \mathrm{GeV}$, corresponding to a centre of mass energy $\sqrt{s}=319 \mathrm{GeV}$. The luminosity amounts to $22 \mathrm{pb}^{-1}$. The measurements are combined with similar data taken in $1996 / 1997$ at $E_{p}=$ $820 \mathrm{GeV}$ [1]. The present paper refers to methods and detailed explanations given in a recent publication [2] of lower $Q^{2}$ data, $0.2 \mathrm{GeV}^{2} \leq Q^{2} \leq 12 \mathrm{GeV}^{2}$. The results can be compared with data obtained by the ZEUS Collaboration $[3,4]$.

The double differential neutral current DIS cross section in its reduced form and neglecting contributions from $Z$ boson exchange, is given by

$$
\begin{aligned}
\sigma_{r} & =\frac{Q^{4} x}{2 \pi \alpha^{2}\left[1+(1-y)^{2}\right]} \cdot \frac{\mathrm{d}^{2} \sigma}{\mathrm{d} x d Q^{2}} \\
& =F_{2}\left(x, Q^{2}\right)-f(y) \cdot F_{L}\left(x, Q^{2}\right)
\end{aligned}
$$

with $\alpha$ denoting the fine structure constant and $f(y)=$ $y^{2} /\left[1+(1-y)^{2}\right]$. The inelasticity $y$ is related to $Q^{2}, x$ and the centre-of-mass energy squared, $s=4 E_{e} E_{p}$, by $y=$ $Q^{2} / s x$. A first measurement of the longitudinal structure function $F_{L}$ at low $x$ was recently performed by $\mathrm{H} 1$ [5]. The measurement presented here is restricted to the region of inelasticity $y \leq 0.6$ where the contribution of $F_{L}$ is small and thus it focuses on the structure function $F_{2}$. The data are used to determine the derivative $\left(\partial F_{2} / \partial \ln Q^{2}\right)_{x}$ which provides a sensitive test of the evolution dynamics of partons, and the derivative $\left(\partial \ln F_{2} / \partial \ln x\right)_{Q^{2}}$, which quantifies the rise of $F_{2}\left(x, Q^{2}\right)$ at fixed $Q^{2}$ towards low $x$ [6].

The new data cover the $Q^{2}$ region of deep inelastic scattering, from a few $\mathrm{GeV}^{2}$ to about $150 \mathrm{GeV}^{2}$, with unprecedented accuracy at low $x$. A QCD analysis at next-to-leading order (NLO) is performed to obtain a new set of parton distribution functions (PDFs) from the inclusive DIS cross section measurements of the $\mathrm{H} 1$ experiment alone. The $\mathrm{QCD}$ analysis is based on the results presented here, the low $Q^{2}$ data [2], and the neutral and charged current (NC and $\mathrm{CC}$ ) data sets at high $Q^{2}[7-9]$.

\section{Measurement technique}

The analysis techniques are similar to those applied at lower $Q^{2}$ [2], more details of the present analysis can also be found in [10].

\subsection{Detector}

The $\mathrm{H} 1$ detector $[11,12]$ was built and upgraded for the accurate measurement of inelastic ep interactions at HERA. The detector components most relevant to this measurement are the central tracker, the backward ${ }^{1}$ lead-scintillator calorimeter (SpaCal) [13] and the liquid argon calorimeter (LAr) [14]. The central tracker consists of the central jet drift chamber, two complementary $z$ drift chambers, the central inner (CIP) $[15,16]$ and outer proportional chambers, and the central silicon tracker (CST) [17]. The drift chambers and the CST are used for the measurement of tracks from the hadronic final state. The momenta of the tracks are determined and the event vertex is reconstructed. The polar angle of the scattered positron is determined by the planar backward drift chamber (BDC) and the vertex position. Complementary tracking information is obtained from the backward silicon tracker (BST) [18]. The SpaCal contains electromagnetic and hadronic sections. Its energy resolution for electromagnetic energy depositions is $\delta E / E \approx 0.07 / \sqrt{E / \mathrm{GeV}} \oplus 0.01$. It also provides a trigger based on the scattered positron energy. The LAr allows the hadronic final state to be reconstructed. Its energy resolution was determined to be $\delta E / E \approx 0.50 / \sqrt{E / \mathrm{GeV}} \oplus$ 0.02 with pion test beam data [19]. Two electromagnetic crystal calorimeters, a photon tagger and an electron tagger, located close to the beam pipe at $z=-103.1 \mathrm{~m}$ and

\footnotetext{
${ }^{1}$ The backward direction is determined by the outgoing positron beam direction. H1 uses a coordinate system with the positive $z$ axis given by the outgoing proton beam direction and the nominal interaction point at $z=0$.
} 
$z=-33 \mathrm{~m}$, respectively, are used to monitor the luminosity via the measurement of the Bethe-Heitler process ep $\rightarrow$ rep.

\subsection{Online event selection}

The online trigger conditions used in this analysis are based on an energy deposition in the electromagnetic section of the SpaCal. Three trigger conditions with different energy and radius thresholds are used, which largely overlap in phase space. The dominant source of trigger inefficiency are the veto conditions against beam related background. For these a global inefficiency of $(0.5 \pm 0.3) \%$ is determined and corrected for. The inefficiency of the online software filter is determined to be $0.2 \%$, which is applied as a global correction with a systematic uncertainty of half that size. The residual trigger inefficiencies from other sources are smaller than $0.1 \%$.

\subsection{Kinematics}

The DIS event kinematics are reconstructed from scattered positron and hadronic final state information. The positron energy $E_{e}^{\prime}$ and scattering angle $\theta_{e}$ are used. For the hadronic final state a sum over the particles' energies $E_{i}$ and longitudinal momenta $P_{z, i}$ is formed, $\Sigma_{h}=\sum_{i}\left(E_{i}-P_{z, i}\right)$. The total difference between energy and longitudinal momentum $E-P_{z}$ is obtained by adding to $\Sigma_{h}$ the positron contribution, $E-P_{z}=\Sigma_{h}+E_{e}^{\prime}\left(1-\cos \theta_{e}\right)$. Based on these variables, measurements of $Q^{2}$ and $y$ are obtained using the electron and the $\Sigma$ methods as explained in [2]. In order to optimise the measurement accuracy, the electron method is used at larger $y \gtrsim 0.1$, while the $\Sigma$ method is used at lower $y$. For this analysis, positrons with scattering angles between $\theta_{e} \approx 158^{\circ}$ and $\theta_{e} \approx 173^{\circ}$, energies $E_{e}^{\prime}>11 \mathrm{GeV}$ and $Q_{e}^{2}>10 \mathrm{GeV}^{2}$ are included. The cross section measurement is performed in bins of $x$ and $Q^{2}$ chosen similarly to the previous measurement [1], with small modifications due to the different centre of mass energy. For each bin and reconstruction method the purity $P=N_{\text {rec,gen }} / N_{\text {rec }}$ and the stability $S=N_{\text {rec,gen }} / N_{\text {gen }}$ are calculated. Here $N_{\text {rec }}\left(N_{\text {gen }}\right)$ is the total number of reconstructed (generated) Monte Carlo events in the bin and $N_{\text {rec,gen }}$ is the number of events which are both generated and reconstructed in the same bin. The values for purity and stability exceed $40 \%$ in all analysis bins and are typically well above $50 \%$ for the chosen reconstruction method [10].

\subsection{Positron and hadronic final state reconstruction}

The reconstruction of the scattered positron is based on the measurement of a deposition of energy, termed a cluster, with a limited transverse size characteristic of an electromagnetic shower. The energy of the cluster $E_{e}^{\prime}$ is obtained by summing over all cells of the cluster in the electromagnetic section of the SpaCal. Its transverse size is characterised by $R_{\log }$, which is obtained from the positions of all SpaCal cells belonging to a cluster using a logarithmic energy weighting [20]. A cut $R_{\log }<4 \mathrm{~cm}$ is applied. For additional background suppression, the energy deposition in the hadronic section of the SpaCal behind the electromagnetic cluster, $E_{\mathrm{h}}$, is required to be less than $15 \%$ of $E_{e}^{\prime}$. The positron candidate cluster is further required to be associated to a track in the BDC, formed by at least 4 hits from the 8 layers. This ensures an accurate measurement of the polar angle $\theta_{e}$ in combination with the $z$ position of the interaction vertex, $z_{\mathrm{vtx}}$, determined with the central track detectors.

The reconstruction of the hadronic final state uses information from the central tracker and the calorimeters LAr and SpaCal [2]. The determination of $\Sigma_{h}$ is affected by the presence of noise in the calorimeters. The resulting bias is particularly strong for small $y_{h}=\Sigma_{h} / 2 E_{e}$. Contributions of noise from the SpaCal and the LAr are suppressed as described in [2].

\subsection{Monte Carlo event simulations}

Monte Carlo (MC) simulations are used to correct for detector acceptance and resolution effects, and for the background subtraction. The DIS signal events are generated using the DJANGOH [21] event generator, while the PHOJET [22] program is used for the photoproduction background. Elastic QED Compton events are generated using the COMPTON event generator [23]. The cross section measurement is corrected for QED radiation up to order $\alpha$ using HERACLES [24]. The radiative corrections are cross checked with HECTOR [25]. An agreement to better than $0.3 \%$ is found in the kinematic range of this measurement.

All generated events are passed through the full GEANT [26] based simulation of the H1 apparatus and are reconstructed using the same program chain as for the data. For consistency, the calibrations of the SpaCal and the LAr, as well as the alignment, are performed for the reconstructed $\mathrm{MC}$ events in the same way as for the data. The calorimeter noise is determined using events from random triggers and is overlaid on the simulated events. The simulated events are reweighted to match the cross section derived from the QCD fit which is described in Sect. 6.

\section{Data analysis}

The data for the present measurement were recorded in the year 2000 and correspond to an integrated luminosity of $22 \mathrm{pb}^{-1}$. In the following a description of the analysis is given. Further information can be found in $[2,10]$. 


\subsection{Event selection}

An overview of the selection criteria is given in Table 1. Detection of the scattered positron is required within the backward calorimeter SpaCal, as discussed in Sects. 2.3 and 2.4. In order to ensure that the cluster is well contained in the $\mathrm{SpaCal}$, the extrapolation of the associated BDC track segment to the SpaCal plane is required to be within a distance from the beam line of $r_{\text {Spac }}<73 \mathrm{~cm}$. The event vertex is reconstructed either by the central drift chambers and the CST or by using the CIP and the positron cluster position. Its $z$ position is required to be within $\pm 35 \mathrm{~cm}$ of the centre of the detector.

Events for which the hadronic final state is poorly reconstructed are rejected by demanding that the total measured hadronic transverse momentum $P_{\mathrm{T}}^{h}$ be at least $30 \%$ of the positron transverse momentum $P_{\mathrm{T}}^{e}$. This efficiently removes migrations from very low $y$. Events with high energy initial state photon radiation are excluded by requiring $E-P_{z}>35 \mathrm{GeV}$. The QED Compton process is suppressed using a topological cut against events with two back-to-back electromagnetic clusters reconstructed in the SpaCal.

\subsection{Efficiency determination}

The efficiencies of the positron identification requirements (transverse cluster size, hadronic energy fraction, BDC validation) exceed $99 \%$ in most of the phase space. They are well described by the simulation. The only significant local inefficiency, of about $5 \%$, is observed for the BDC validation requirement at a radial distance from the beam line of $r_{\mathrm{BDC}} \sim 25 \mathrm{~cm}$ where the geometry of the BDC drift cells changes. A detailed map of this inefficiency as a function of $r_{\mathrm{BDC}}$ and the azimuthal angle of the scattered positron is obtained using positron candidates validated by the BST. The simulation is adjusted accordingly and an additional

Table 1 Event selection criteria

\begin{tabular}{ll}
\hline Description & Requirement \\
\hline Kinematic Range & $Q_{e}^{2}>10 \mathrm{GeV}^{2}$ \\
Scattered positron energy & $E_{e}^{\prime}>11 \mathrm{GeV}$ \\
SpaCal cluster radius & $R_{\mathrm{log}}<4 \mathrm{~cm}$ \\
Energy in hadronic SpaCal section & $E_{\mathrm{h}} / E_{e}^{\prime}<0.15$ \\
BDC validation & $\geq 4$ linked hits, BDC-SpaCal \\
& radial match $<2.5 \mathrm{~cm}$ \\
Radial cluster position & $r_{\mathrm{Spac}}<73 \mathrm{~cm}$ \\
Vertex $z$ position & $\left|z_{\mathrm{vtx}}\right|<35 \mathrm{~cm}$ \\
Transverse momentum balance & $P_{\mathrm{T}}^{h} / P_{\mathrm{T}}^{e}>0.3$ \\
Longitudinal momentum balance & $E-P_{z}>35 \mathrm{GeV}$ \\
QED Compton Rejection & Topological veto \\
\hline
\end{tabular}

$0.5 \%$ systematic uncertainty is added to the cross section measurement error, uncorrelated from bin-to-bin.

The efficiency of the vertex reconstruction is determined using events independently reconstructed by the BST. This efficiency is determined to be close to $100 \%$ for all but a few bins at $Q^{2}<20 \mathrm{GeV}^{2}$ and $y<0.03$, where it drops to about 75-95\%. It is described by the simulation to an accuracy of $0.3 \%$ for $y>0.01$. For lower $y$ the description is accurate to about $1 \%$, which is accounted for by an additional uncorrelated uncertainty in the corresponding bins.

\subsection{Alignment and calibration}

The alignment of the $\mathrm{H} 1$ detector starts from the internal adjustment of the central tracker and proceeds with the backward detectors, BDC, SpaCal and BST. The alignment of the BDC is performed using the tracks of positron candidates that are reconstructed in the central tracker with high accuracy. The SpaCal position is adjusted based on the positron tracks measured in the BDC. Finally the BST is aligned using events with a well reconstructed central vertex and a positron track measured in the BDC. The resulting agreement of the polar angle measurements is better than $0.2 \mathrm{mrad}$, which defines the associated systematic uncertainty. The measured and simulated scattered positron polar angle distributions are shown in Fig. 1(a). The data are well described by the simulation.

The calibration of the electromagnetic scale of the $\mathrm{SpaCal}$ corrects for differences in the gain factors of individual SpaCal cells, for local non-uniformities at the sub-cell level and for global non-linearity in the energy response. The calibration is based mainly on the positron candidates at low $y$. For these the kinematics can be reliably reconstructed using the double angle reconstruction method as described in [2], which employs only the polar angle information of the hadronic final state and the scattered positron, in addition to the positron beam energy. The non-linearity of the energy response is determined and corrected for using a sample of $\pi^{0} \rightarrow \gamma \gamma$ events. The energy scale is then checked using elastically produced $J / \psi$ particles decaying to $e^{+} e^{-}$and QED Compton events, $e p \rightarrow e p \gamma$, where the scattered positron and photon are both reconstructed in the SpaCal. The relative data-to-simulation scale uncertainty is derived from two contributions: a global $0.2 \%$ arising from the double angle calibration and a part resulting from the $\pi^{0}$ studies, which is $1 \%$ at $E=2 \mathrm{GeV}$ linearly decreasing to zero at $E=27.6 \mathrm{GeV}$. The measured and simulated scattered positron energy distributions are in very good agreement, as shown in Fig. 1(b).

The calibration of the calorimeters employed for the hadronic final state energy measurement is based on kinematic constraints relating the scattered positron to the hadronic final state. For the calibration of the LAr calorimeter, the conservation of the total transverse momentum $P_{\mathrm{T}}$ is 
Fig. 1 Distribution of events requiring $y_{e}<0.6$ and $y_{\Sigma}>0.005$ : the polar angle (a) and the energy (b) of the scattered positron, the transverse momentum ratio $P_{\mathrm{T}}^{h} / P_{\mathrm{T}}^{e}$ (data outside the analysis selection indicated by the vertical line shown as open symbols) (c) and $E-P_{z}(\mathbf{d})$. The curves represent the MC simulation normalised to the luminosity, including a very small contribution from photoproduction. The narrow bands illustrate the correlated systematic uncertainties of the measurement
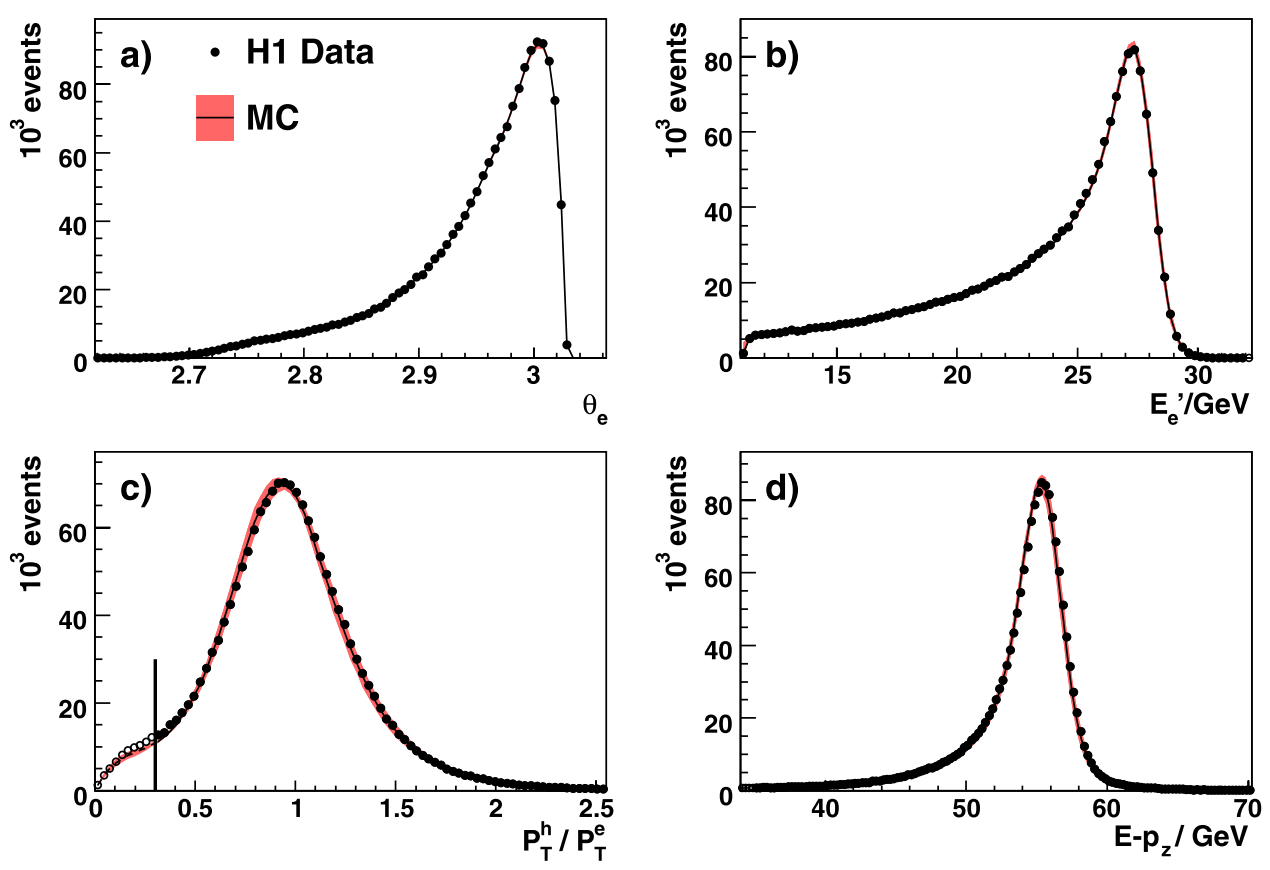

used. The hadronic SpaCal calibration utilises the conservation of $E-P_{z}$.

The transverse momentum balance between the scattered positron and the hadronic final state is studied as a function of various variables, such as $P_{\mathrm{T}}^{e}$, the polar angle of the hadronic final state, and $y_{\Sigma}$. Figure 1(c) shows the overall $P_{\mathrm{T}}^{h} / P_{\mathrm{T}}^{e}$ distribution with a vertical line at 0.3 indicating the analysis cut value. For $P_{\mathrm{T}}^{h} / P_{\mathrm{T}}^{e}$ values larger than this cut, the data distribution lies inside a band given by varying the LAr hadronic energy scale in the simulation by $2 \%$ for $y>10^{-2}$. At the lowest $y \simeq 0.005$ considered in the measurement, the hadronic final state is produced at small polar angles, and a significant part escapes detection. The systematic uncertainty on the hadronic energy scale is increased from $2 \%$ at $y=10^{-2}$ linearly in $\log y$ to $10 \%$ at $y=10^{-3}$. Furthermore, for bins with $y<10^{-2}$, an additional uncorrelated cross section uncertainty of $2 \%$ is estimated by varying the $P_{\mathrm{T}}^{h} / P_{\mathrm{T}}^{e}$ cut between 0.25 and 0.35 .

A topological finder is used to identify and subtract LAr noise. The fraction of hadronic energy attributed to noise is described by the simulation to within $15 \%$, which is taken as a systematic uncertainty.

For large values of $y$, the contribution of the SpaCal to $\Sigma_{h}$ becomes larger than the combined contribution of the LAr calorimeter and the tracks, and thus the total $E-P_{z}$, expected to be $2 E_{e}$, provides the calibration for the hadronic final state measurement in the SpaCal. A study at $y \simeq 0.5$ shows that the hadronic energy measurement in the SpaCal is described by the simulation to $0.3 \mathrm{GeV}$. Figure 1 (d) shows the $E-P_{z}$ distribution for the data and the simulation. The simulation reproduces the data within the combined calibration uncertainties.

\subsection{Background}

A small source of background for this analysis arises from photoproduction events, in which the scattered positron escapes detection in the backward beam pipe while a particle from the hadronic final state mimics the positron. For a fraction of photoproduction events the scattered positron is detected by the electron tagger of the luminosity system. The photoproduction MC (PHOJET) is normalised globally based on a comparison with tagged events applying all selection criteria apart from the $E-P_{z}$ cut. The systematic uncertainty on the background normalisation is taken to be $15 \%$, based on the studies described in [2]. The background contribution at the highest $y \sim 0.5$ considered here typically amounts to $5 \%$, hence yielding an uncertainty of less than $1 \%$ on the cross section. Potential background from nonep interactions is studied using non-colliding bunches and found to be negligible.

\subsection{Summary of systematic uncertainties}

The systematic uncertainties are classified into two groups, bin-to-bin correlated and uncorrelated errors. They are summarised in Table 2. The electromagnetic and hadronic energy scales, the positron scattering angle, LAr noise, background subtraction and normalisation are all considered to be correlated sources of uncertainty. The uncorrelated errors arise from various efficiencies and the radiative corrections. For most of the analysis phase space none of the sources of systematic uncertainty dominates the result. For very low 
Table 2 Summary of the systematic uncertainties. For the correlated error sources, the uncertainties are given in terms on the uncertainty on the corresponding source. For the uncorrelated error sources, the uncertainties are quoted directly in terms of the effect on the measured cross section

Correlated systematic errors

\begin{tabular}{ll}
\hline Source & Uncertainty \\
\hline$E_{e}^{\prime}$ scale & $0.2 \%$ \\
$E_{e}^{\prime}$ linearity & $1 \%$ at $2 \mathrm{GeV}$ to $0 \%$ at $27.6 \mathrm{GeV}$ \\
Polar angle $\theta_{e}$ & $0.2 \mathrm{mrad}$ \\
LAr hadronic scale & $2 \%$ for $y>0.01$ \\
& increasing linearly in log $y$ to $10 \%$ at $y=0.001$ \\
LAr noise contribution to $E-P_{z}$ & $15 \%$ \\
SpaCal hadronic scale & $0.3 \mathrm{GeV}$ \\
$\gamma p$ background normalisation & $15 \%$ \\
Luminosity and other global uncertainties & $1.2 \%$
\end{tabular}

Uncorrelated systematic errors

\begin{tabular}{ll}
\hline Source & Uncertainty \\
\hline BDC efficiency & $0.5 \%$ \\
Vertex efficiency & $0.3 \%$ \\
Radiative corrections & $0.3 \%$ \\
Additional uncertainty for bins with $y \leq 0.01$ & $2.0 \%$ \\
\hline
\end{tabular}

Fig. 2 Bjorken- $x$ and $Q^{2}$ distributions of events requiring $y_{e}<0.6$ and $y_{\Sigma}>0.005: x_{e}$ and $Q_{e}^{2}$ reconstructed using the electron method (a)-(b), and $x_{\Sigma}$ and $Q_{\Sigma}^{2}$ reconstructed using the $\Sigma$ method (c)-(d). The curves represent the MC simulation normalised to the luminosity. The narrow bands illustrate the correlated systematic uncertainties of the measurement. The small photoproduction background is drawn shaded
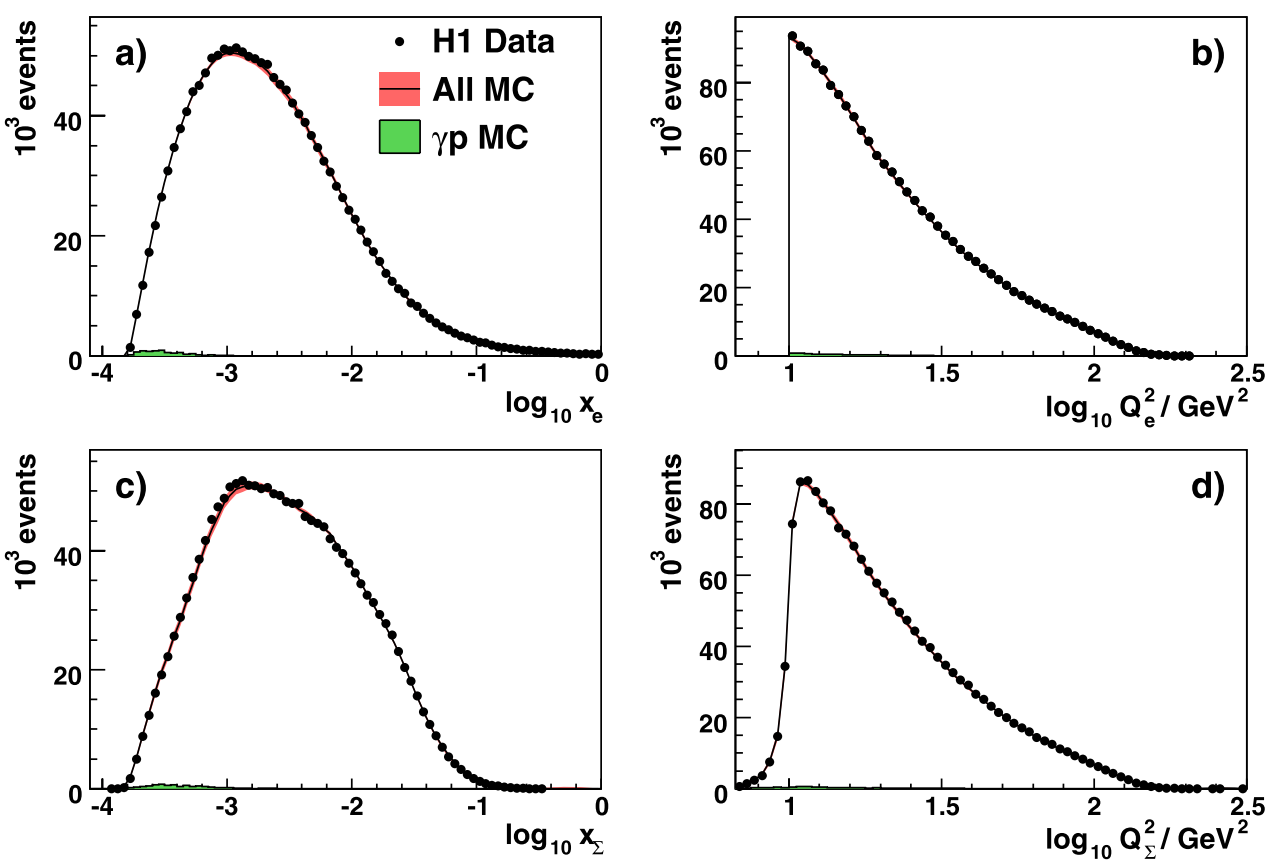

$y<0.01$, many uncertainties increase strongly, as the reconstruction efficiency drops rapidly and the modelling of the hadronic final state suffers from increased losses in the forward direction.

The uncertainty on the global normalisation of the measurement is determined mostly from the luminosity measurement, which is accurate to within $1.1 \%$. The additional corrections discussed in Sect. 2.2 increase the overall normalisation uncertainty to $1.2 \%$.

\subsection{Data-Monte Carlo comparison}

The quality of the MC description of the measurement can be verified by comparing experimental and simulated distributions in the kinematic range of the measurement, corresponding approximately to $y_{\Sigma}>0.005$ and $y_{e}<0.6$. The DIS MC cross section prediction is reweighted to the QCD fit discussed in Sect. 6. The distributions are normalised to the luminosity. Figure 2(a)-(d) shows the $x$ and $Q^{2}$ distribu- 


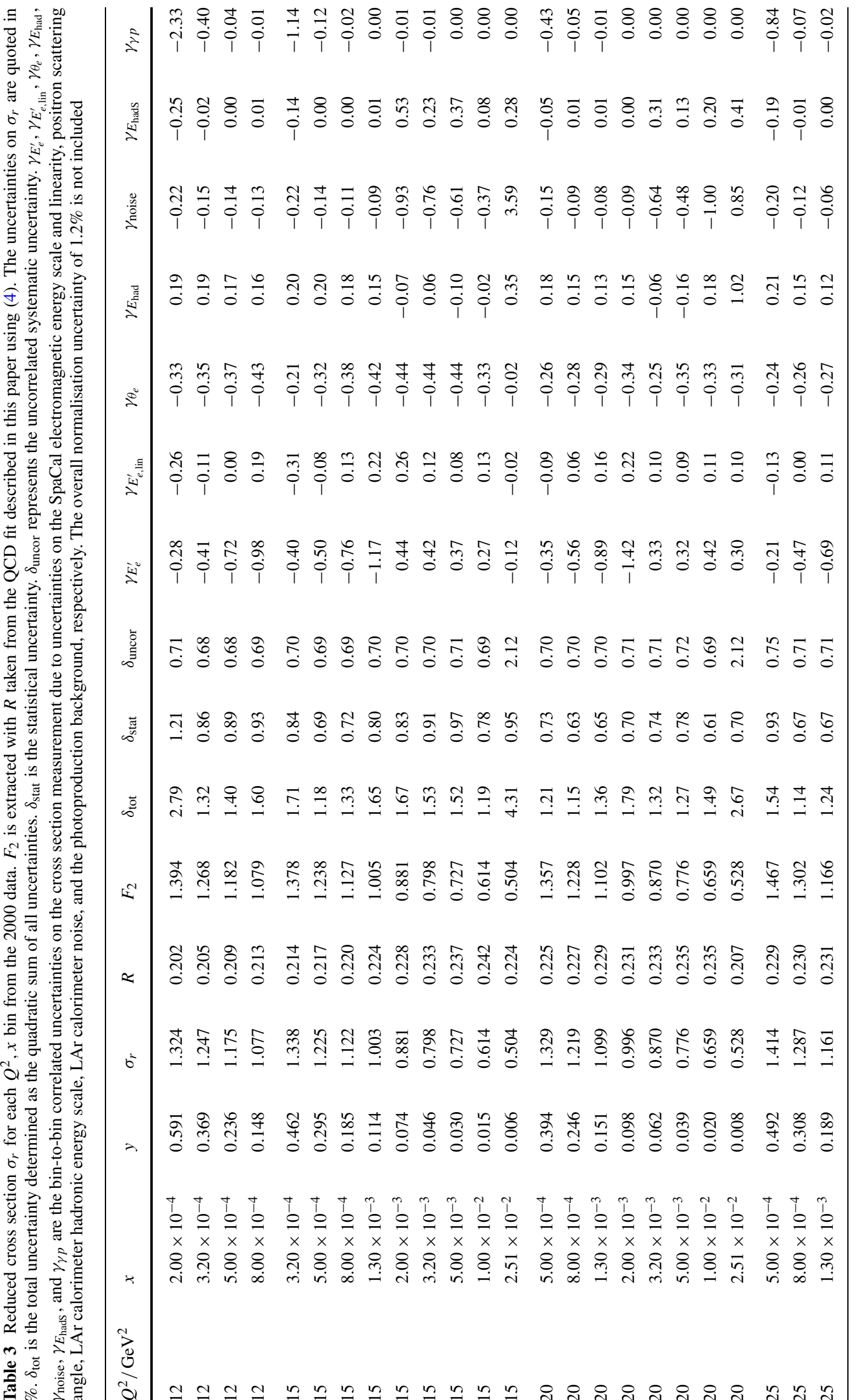




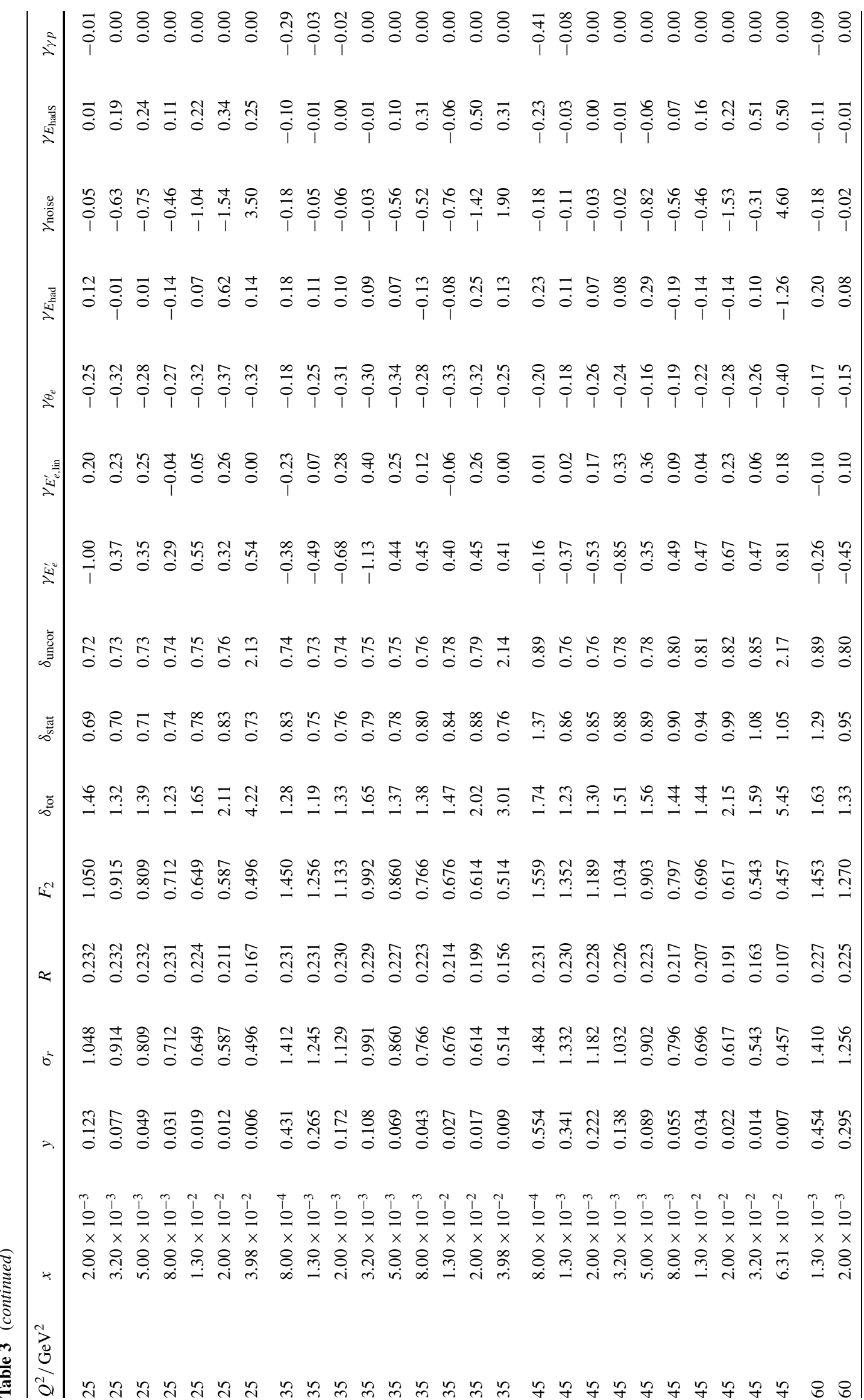




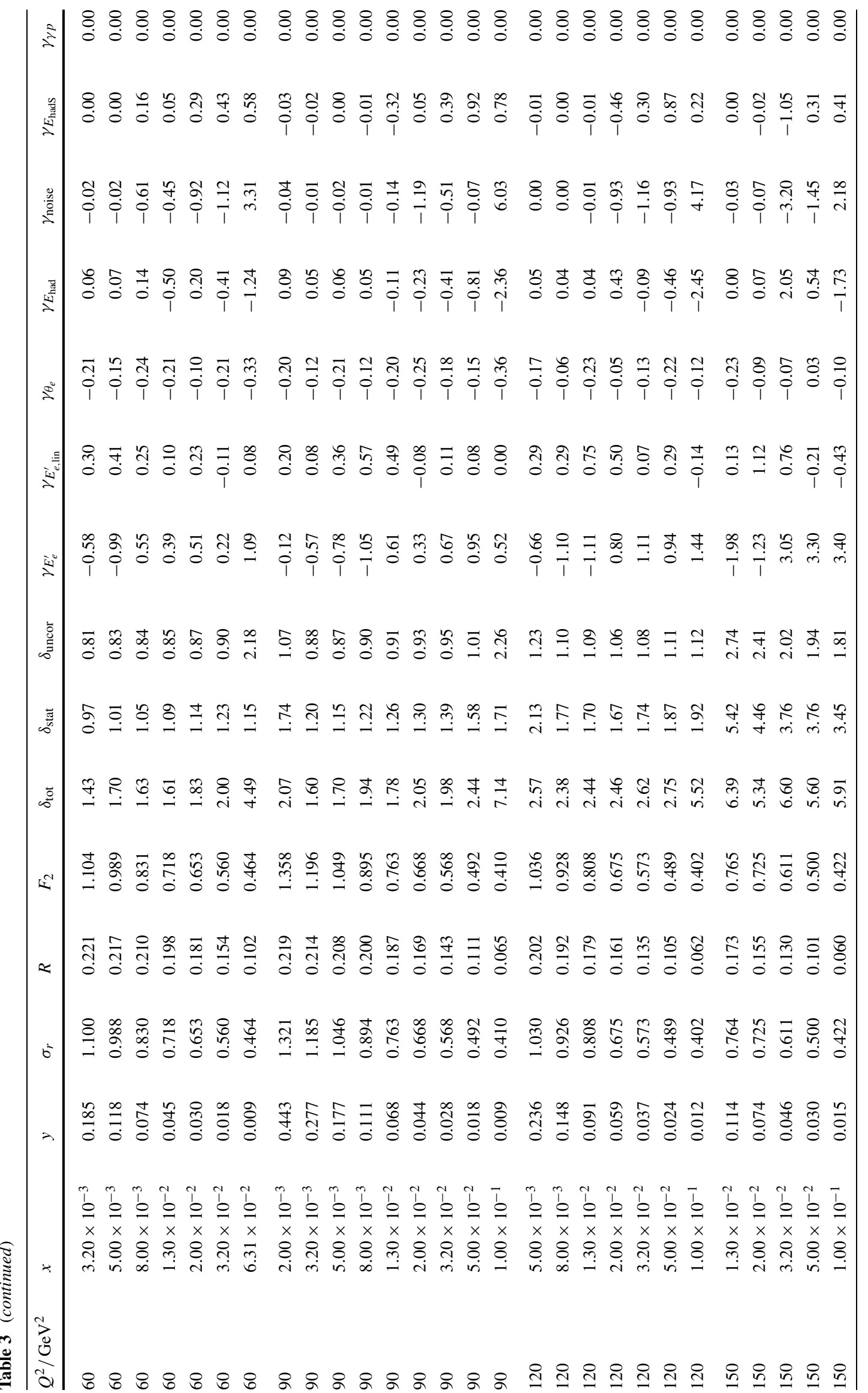


tions of the selected events for the two kinematic reconstruction methods, electron and $\Sigma$. A good overall agreement is obtained in the description of the data by the simulation.

\section{DIS cross section results}

The measurement of the inclusive double differential cross section for deep inelastic positron-proton scattering, $e^{+} p \rightarrow$ $e^{+} X$ with the $E_{p}=920 \mathrm{GeV}$ data, is reported in Table 3 . The table shows the statistical, uncorrelated and the various correlated error contributions. The region of small Bjorken $x, 2 \times 10^{-4} \leq x \leq 0.1$, and of four-momentum transfer squared, $12 \mathrm{GeV}^{2} \leq Q^{2} \leq 150 \mathrm{GeV}^{2}$, is covered.

The stability of the cross section measurement is tested with a set of dedicated cross checks. This is done by splitting the data into two approximately equal subsamples and comparing the results obtained using the same reconstruction method on each. For example, the data are compared as measured with the upper and the lower half of the SpaCal, for negative and positive $z_{\mathrm{vtx}}$ positions, and dividing the sample into early and late data taking periods. These tests are sensitive to local efficiency problems, energy miscalibrations, or the stability of the luminosity measurement. A particularly interesting test is the comparison of the cross section measurements performed with the electron and $\Sigma$ methods, which have different sensitivities to systematic error sources. The results shown in Fig. 3 demonstrate very good agreement taking the correlated uncertainties into account. For the final result, the method with the smaller total uncertainty is chosen in each bin, which results in a transition near $y=0.1$.

This analysis improves the uncertainties by up to a factor of two with respect to previously published results on the inclusive DIS cross section in this kinematic range by $\mathrm{H} 1$ [1] and by ZEUS [3, 4].

\section{Combination of 820 and $920 \mathrm{GeV}$ data}

The present measurement is based on data taken with $920 \mathrm{GeV}$ proton beam energy. A similar data set was obtained in $1996 / 1997$ at $820 \mathrm{GeV}$ and published in [1]. The results were obtained from two different samples, called $A$ and $B$, covering higher $Q^{2} \geq 12 \mathrm{GeV}^{2}$ and lower $Q^{2} \leq 12 \mathrm{GeV}^{2}$ regions, respectively. A comparison of the cross section measurement for sample $A$ with the present analysis revealed a significant deviation, which required a dedicated study as described below.

\subsection{Correction of the $820 \mathrm{GeV}$ data}

For the analysis of the $820 \mathrm{GeV}$ data an older DIS event simulation program version was used. If that program version is used without event weighting, the MC cross section agrees to better than $0.3 \%$ with the HECTOR calculation. However, in order to improve the statistical accuracy of the DIS
Fig. 3 Comparison of reduced cross sections as obtained from the $E_{p}=920 \mathrm{GeV}$ data with the electron (closed circles) and $\Sigma$ (open squares) reconstruction methods. The error bars represent the total measurement uncertainties

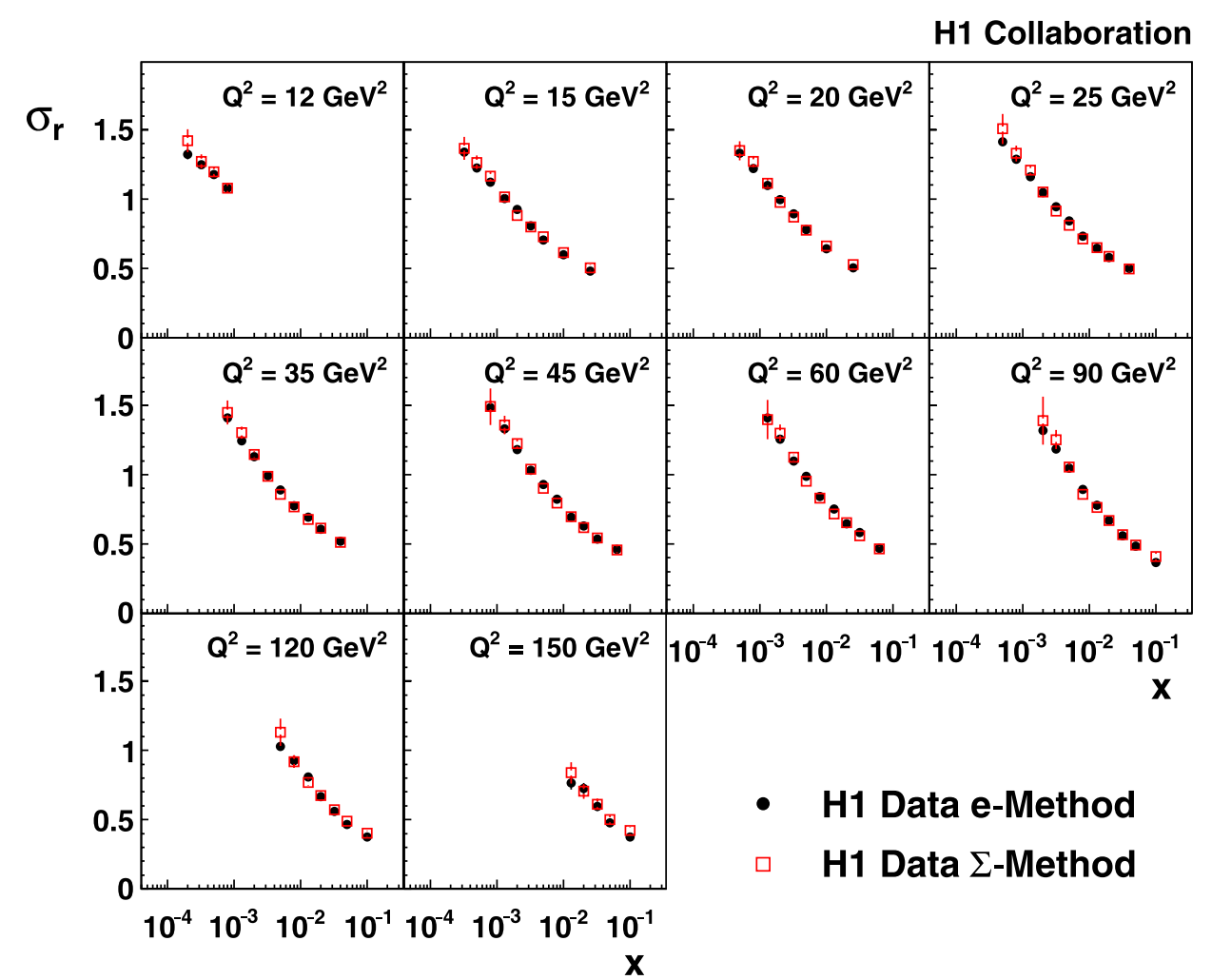


MC event sample, the simulated events in [1] were generated with $Q^{2}$ dependent weights for $Q^{2}<50 \mathrm{GeV}^{2}$. This introduced a bias which depends only on $Q^{2}$. The cross section as obtained in [1] can be corrected by a factor defined as

$c\left(Q^{2}\right)=\frac{N_{\mathrm{MC}}^{\text {weighted }}\left(Q^{2}\right)}{N_{\mathrm{MC}}\left(Q^{2}\right)}$.

Here $N_{\mathrm{MC}}\left(Q^{2}\right)$ and $N_{\mathrm{MC}}^{\text {weighted }}\left(Q^{2}\right)$ are the sums of weights of events generated for a given $Q^{2}$ bin in unweighted and weighted mode, respectively. The dependence of the correction factor on $Q^{2}$ is empirically parameterised by

$c\left(Q^{2}\right)=\left\{\begin{array}{l}c_{0} \quad \text { for } Q^{2}>50 \mathrm{GeV}^{2} \\ c_{0}+c_{1} \cdot \log _{10}\left(Q^{2} / 50 \mathrm{GeV}^{2}\right) \\ \text { for } Q^{2} \leq 50 \mathrm{GeV}^{2}\end{array}\right.$

with $c_{0}=1.027$ and $c_{1}=0.0352$. This correction procedure introduces an additional uncorrelated cross section uncertainty of $0.5 \%$ on the corrected published data.

For a further cross check the 1996/1997 data are reanalysed within the framework of the present analysis. The luminosity determination is improved, resulting in an additional normalisation shift by $+0.5 \%$, corresponding to one third of a standard deviation of the quoted normalisation uncertainty. No further significant deviation is observed between the published results and the reanalysis.

Table 4 presents the 1996/1997 data from [1] corrected for the $Q^{2}$ weighting bias and the small luminosity shift.
This table therefore replaces the previous data for $Q^{2} \geq$ $12 \mathrm{GeV}^{2}$, sample $A$. The measurements of $F_{2}$ for $y<0.6$ are extracted from the corrected cross sections accounting for the $F_{L}$ influence, as described in Sect. 5.3. The sample $B$ in [1], extending to lower values of $Q^{2} \leq 12 \mathrm{GeV}^{2}$, was not affected by the $\mathrm{MC}$ weighting problem and has been combined with further $\mathrm{H} 1$ data as described in [2].

\subsection{Combined cross sections}

The corrected $820 \mathrm{GeV}$ data and the present $920 \mathrm{GeV}$ data are shown in Fig. 4 and combined to determine a new H1 measurement at $12 \mathrm{GeV}^{2} \leq Q^{2} \leq 150 \mathrm{GeV}^{2}$. The combination of the data sets is based on the prescription introduced in [27] and developed further in [2]. The combination uses the reduced cross section data and consistently treats the correlated and uncorrelated uncertainty information, which is given in Tables 3,4 . The data are averaged for the region of large $x$, defined as in [2] by $y<0.35$, but kept separate at higher $y$ where the reduced cross section depends significantly on the centre of mass energy via the $F_{L}$ term. Small differences in the measurement grid are corrected for by adjusting cross section values for points close in $x$ such that they correspond to a common $x$ value, prior to the averaging.

In the combination of the two data sets, assumptions have to be made about the relationship between systematic uncertainty sources, which may not be fully independent between the different analyses of the 1996/1997 and 2000 data. Reasons for correlations between data sets are the similarity in
Fig. 4 Comparison of reduced cross sections as obtained from the $E_{p}=920 \mathrm{GeV}$ (closed circles) and the corrected results from $E_{p}=820 \mathrm{GeV}$ (open squares). The error bars represent the total measurement uncertainties

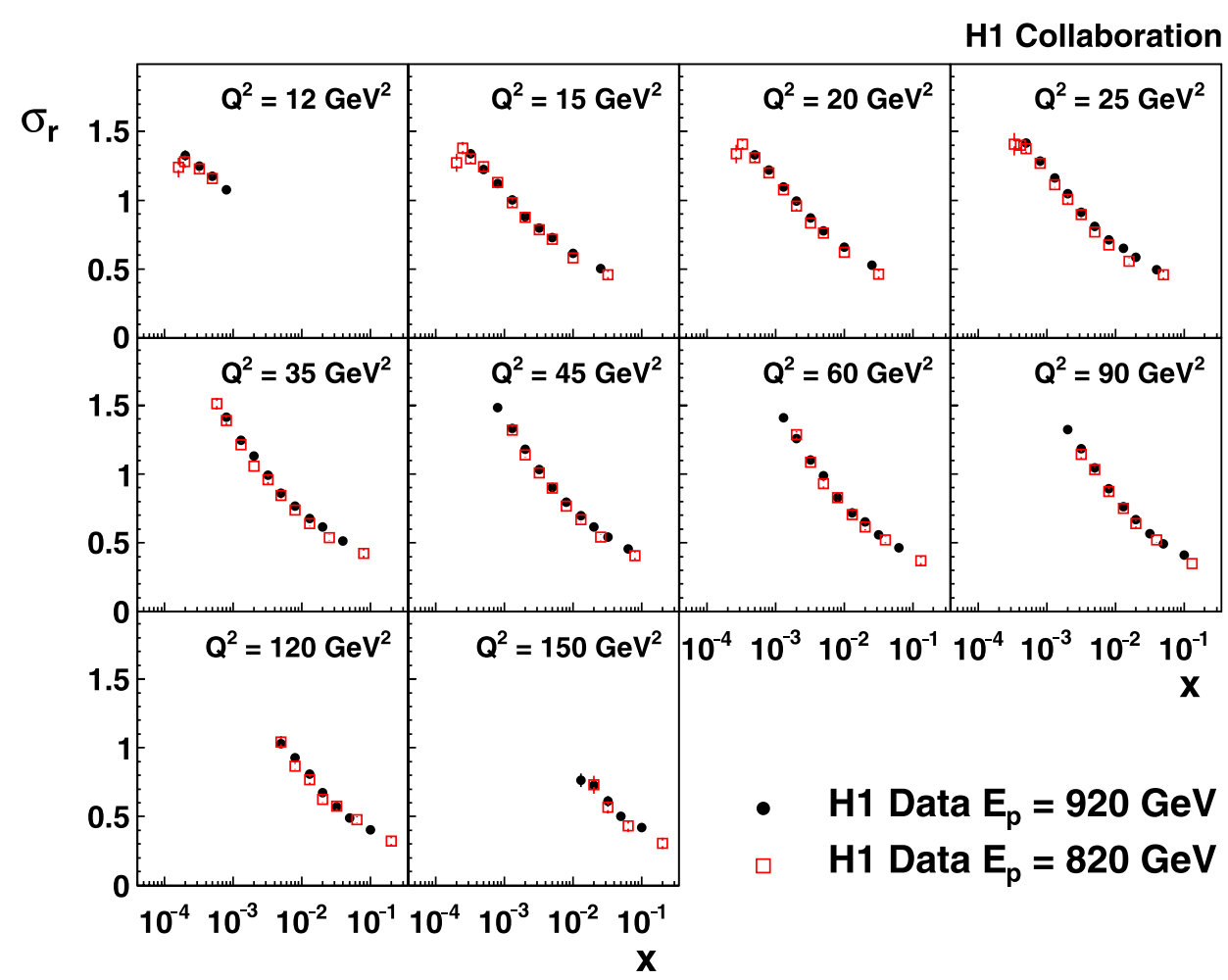




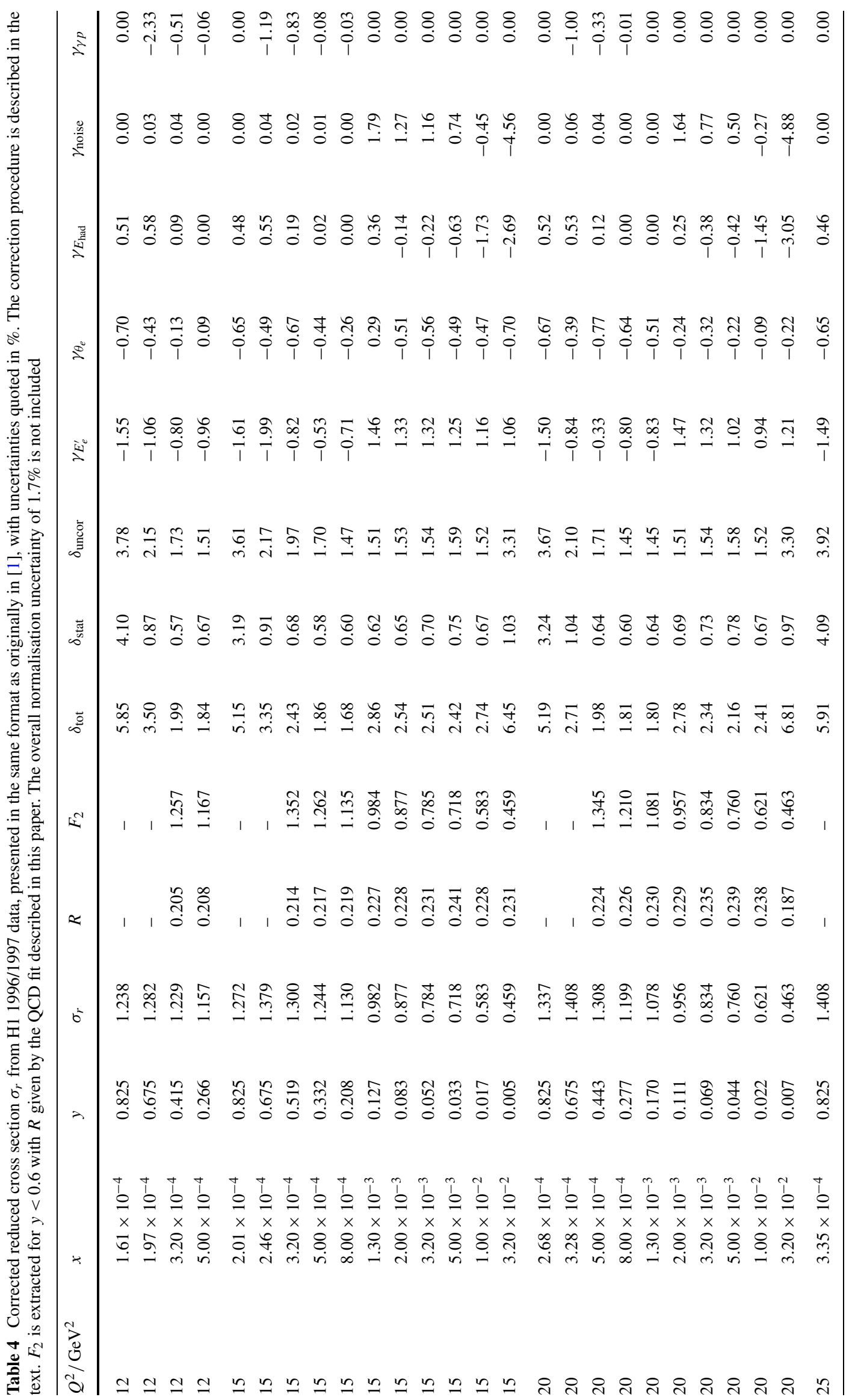




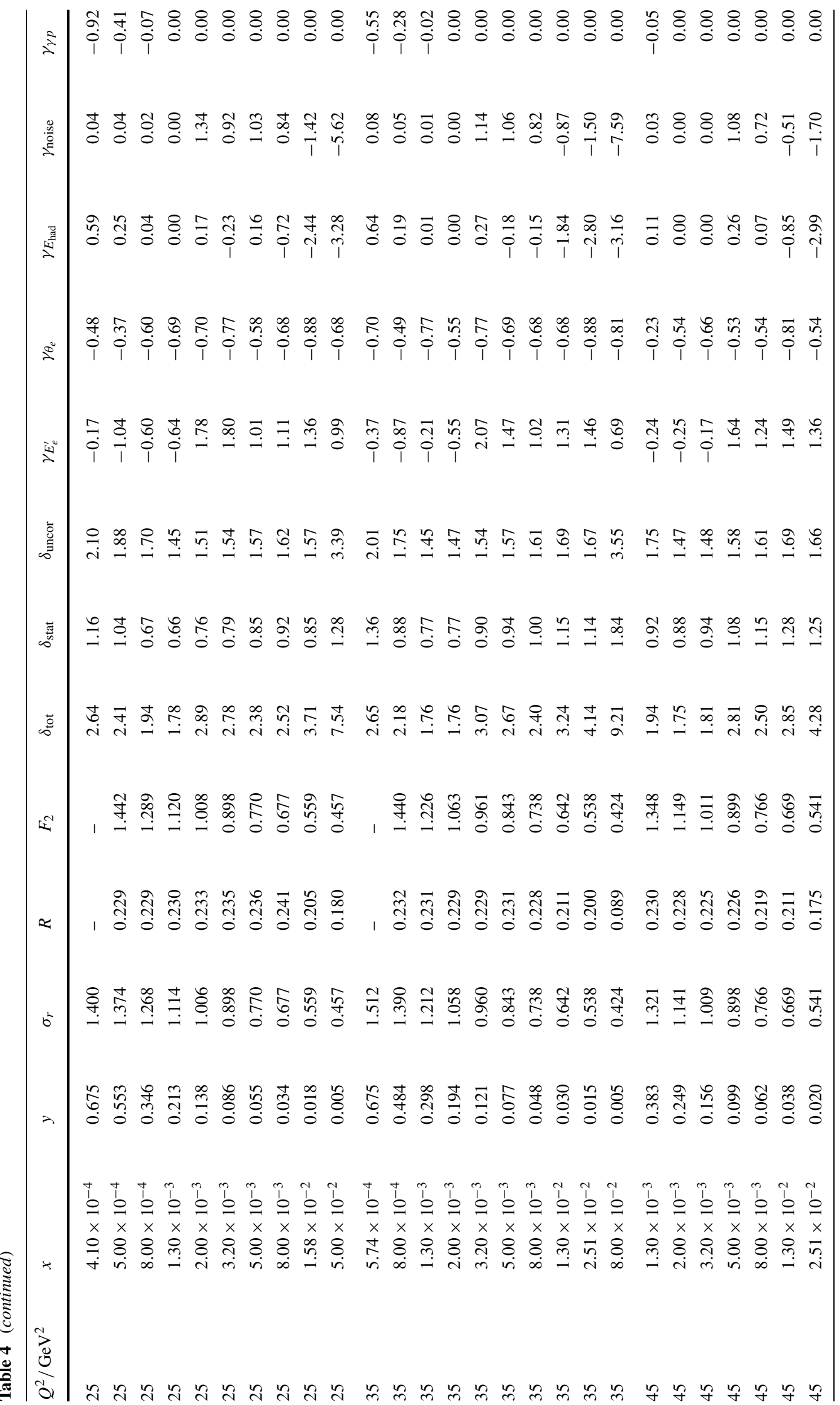




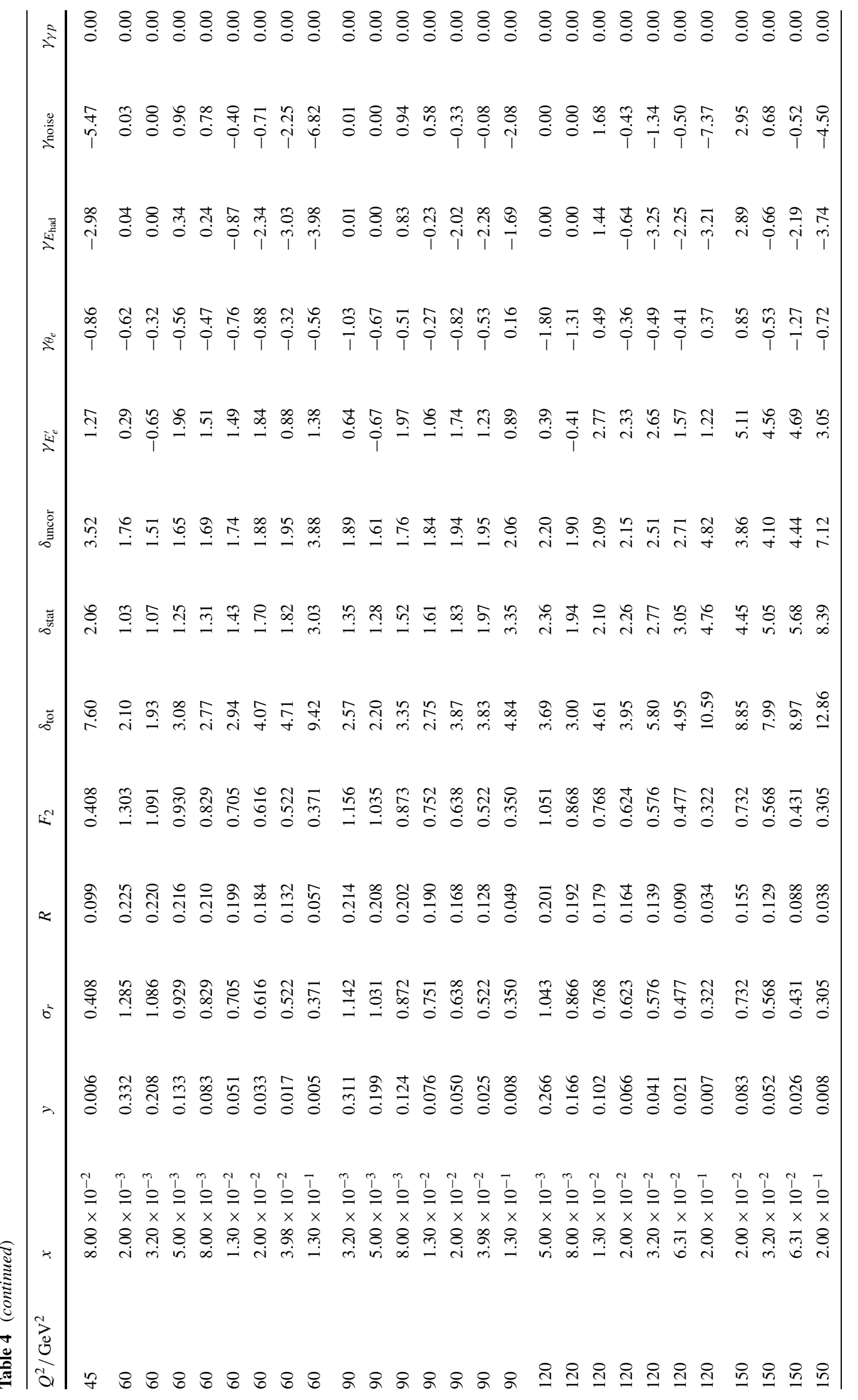


the calibration procedure and the detector setup, whereas uncorrelated effects include variations with time, beam conditions and changes in the analysis procedure. To estimate the sensitivity of the averaged result to the correlation assumptions, different averages of the 1996/1997 and 2000 data are compared, obtained considering all possible assumptions as to which systematic error sources are taken to be correlated or uncorrelated between the data sets. The observed variation of the averaged cross sections is typically at the permille level, which is negligible compared with the total uncertainty. The variations of the total uncertainties never exceed $10 \%$ of the final uncertainty. The assumption that the systematic uncertainties on the two data sets are uncorrelated yields the largest uncertainty. Thus no correlations between the error sources of the 1996/1997 and 2000 data are considered in the combination procedure.

The 1996/1997 and 2000 data sets are fully consistent, as determined in the averaging procedure, with $\chi_{\text {tot }}^{2} / n_{\text {dof }}=$ $51.6 / 61$. As a result of the averaging, the central values of the correlated systematic uncertainties are modified. The shifts of the central values in units of the original uncertainties are given in Table 5. It is observed that none of the absolute values of the shifts exceeds one standard deviation.

The combined measurement of the reduced $e p$ scattering cross section and its uncertainties are listed in Table 6. The correlated uncertainties are given as 14 new sources, $\delta_{i}$, after diagonalisation of the error matrix as explained in [2].
Table 5 Shifts in the central values of the systematic uncertainties determined for the combination of the $1996 / 1997$ data at $E_{p}=820 \mathrm{GeV}$ and the 2000 data at $E_{p}=920 \mathrm{GeV}$. The value for each shift is given in units of the original uncertainty. Note that for the two data sets some of the correlated systematic error sources were defined differently

\begin{tabular}{lcr}
\hline Systematic source & \multicolumn{2}{l}{ Shift } \\
\cline { 2 - 3 } & $1996 / 1997$ & 2000 \\
\hline$E_{e}^{\prime}$ scale & 0.72 & 0.50 \\
$E_{e}^{\prime}$ linearity & - & -0.39 \\
Polar angle $\theta_{e}$ & -0.46 & 0.09 \\
LAr hadronic scale & -0.86 & -0.13 \\
LAr noise & -0.22 & 0.04 \\
SpaCal hadronic scale & - & 0.35 \\
$\gamma p$ background & 0.11 & -0.11 \\
Luminosity & 0.64 & -0.46 \\
\hline
\end{tabular}

The combined $\mathrm{H} 1$ data on the inclusive cross section measurement have total uncertainties of $1.3-2 \%$ in most of the phase space.

\section{$5.3 \quad F_{2}$ and its derivatives}

The cross section data are used to extract $F_{2}$ for $y<0.6$, correcting for the small influence of $F_{L}$ using

$$
F_{2}=\frac{\sigma_{r}}{1-f(y) \frac{R}{1+R}} .
$$

Fig. 5 Measurement of the structure function $F_{2}$ at fixed $Q^{2}$ as a function of $x$. The data of this measurement (closed circles) are complemented by the previously published data at low $Q^{2}$ (open circles) [2] and high $Q^{2}$ (open boxes) [9]. The error bars represent the total measurement uncertainties. The curve represents the QCD fit described in this paper

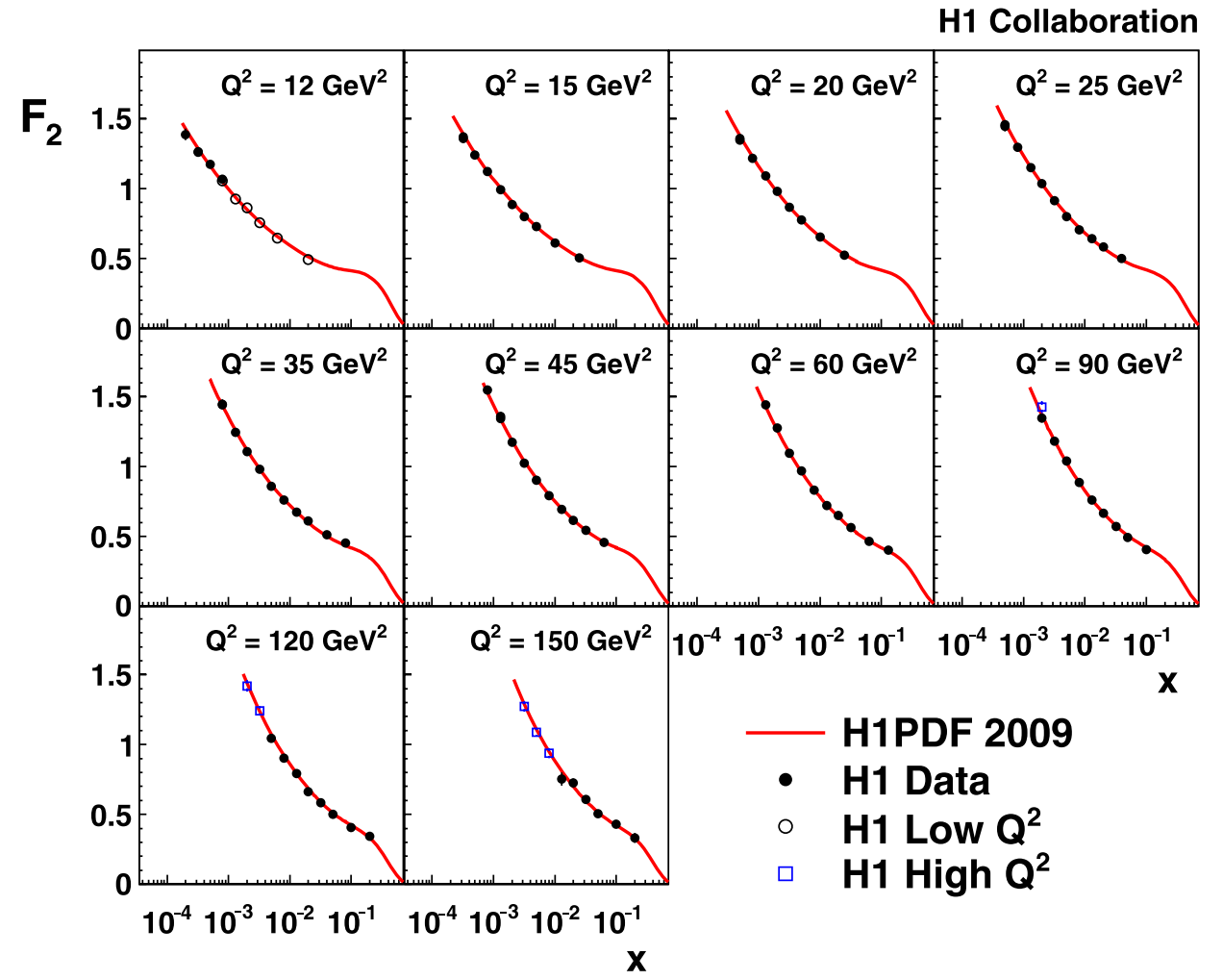




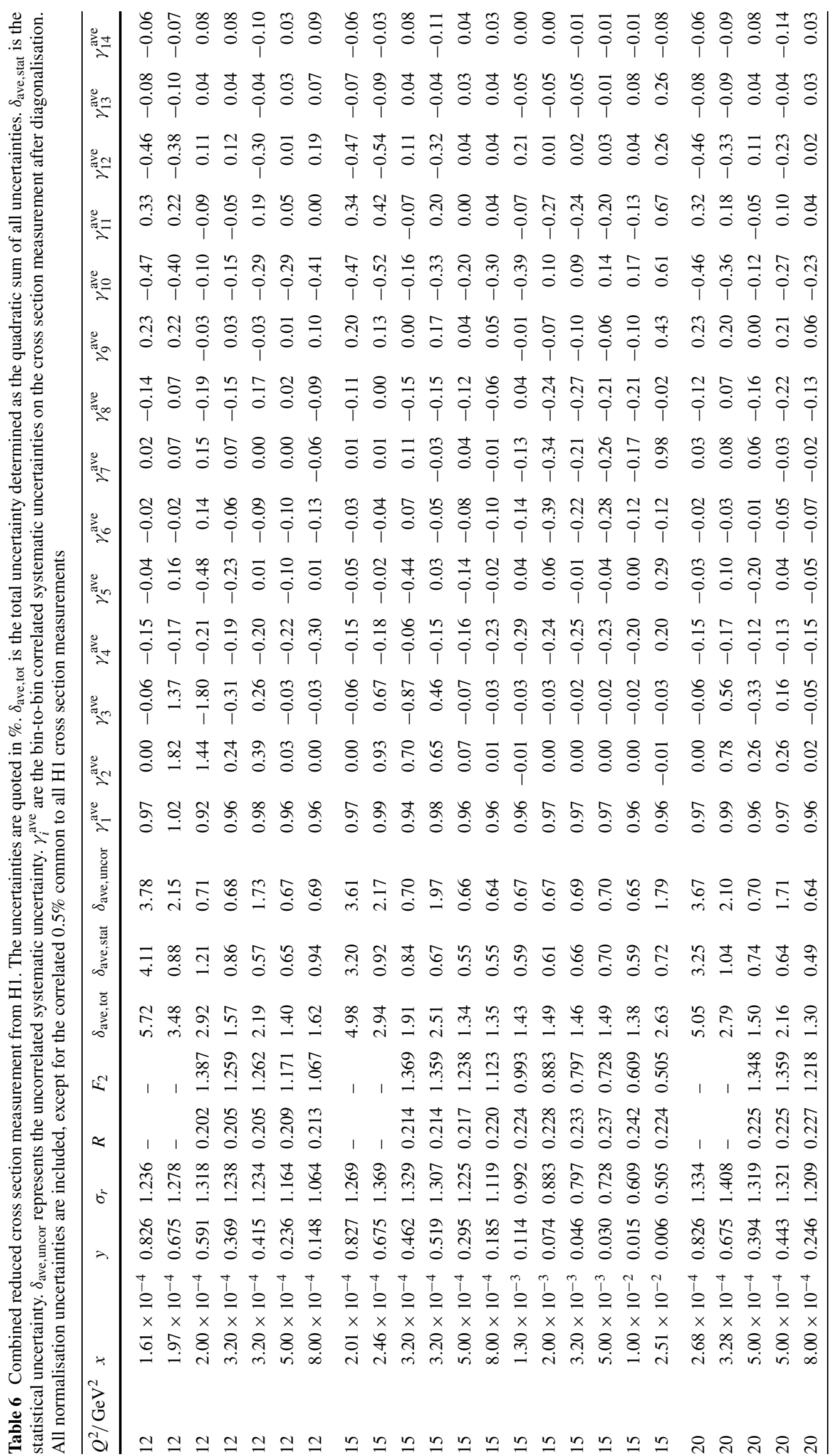




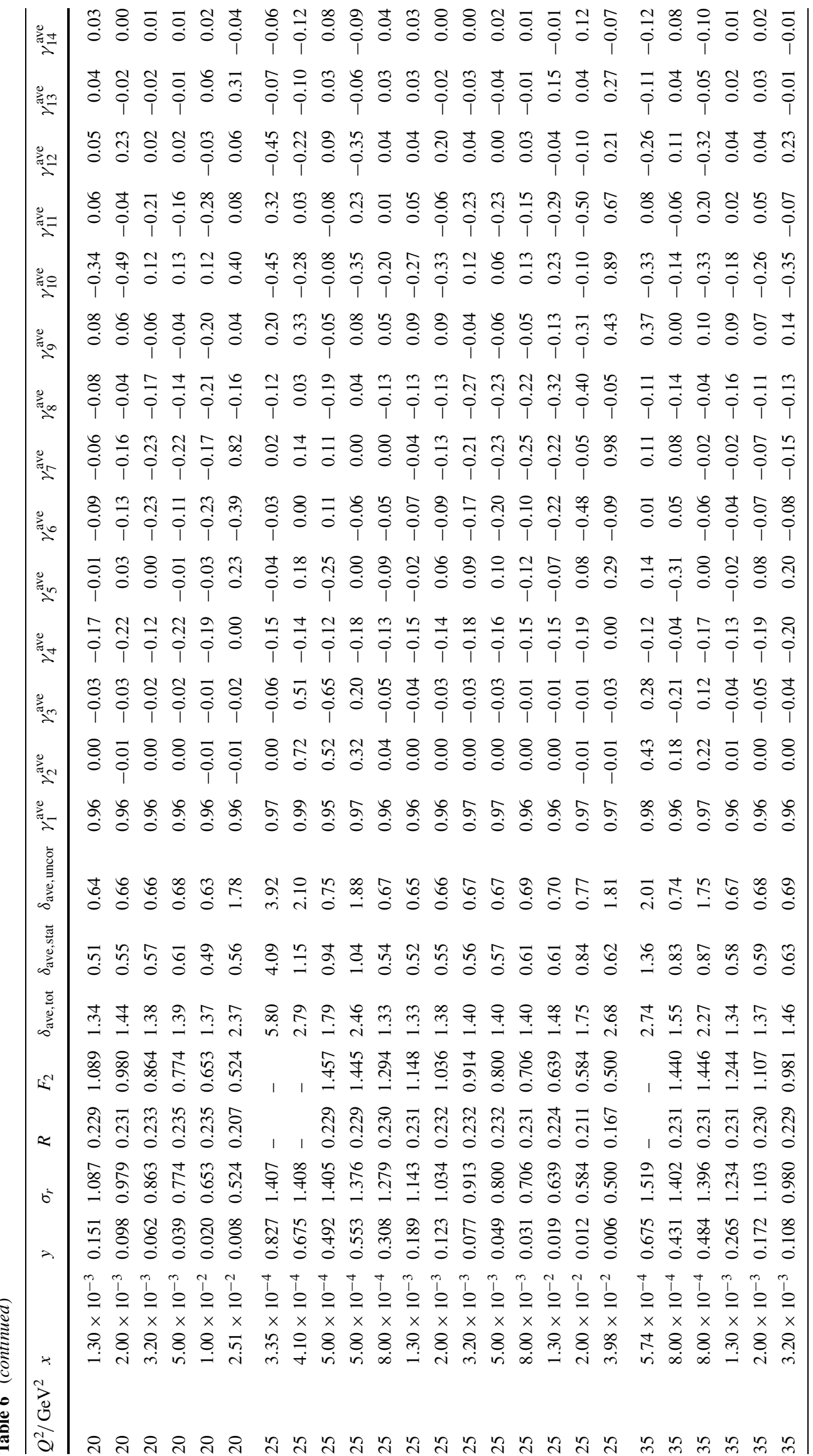




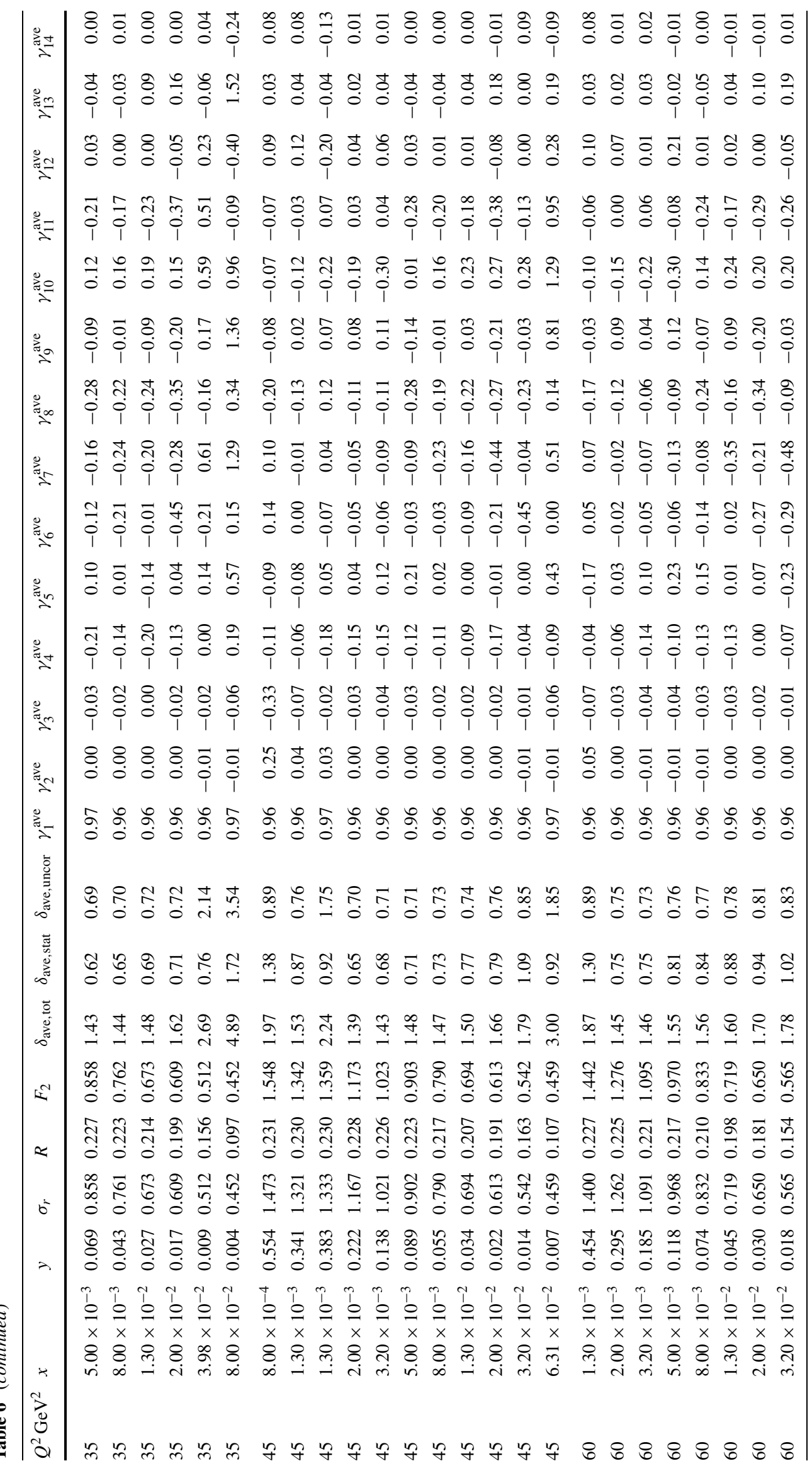




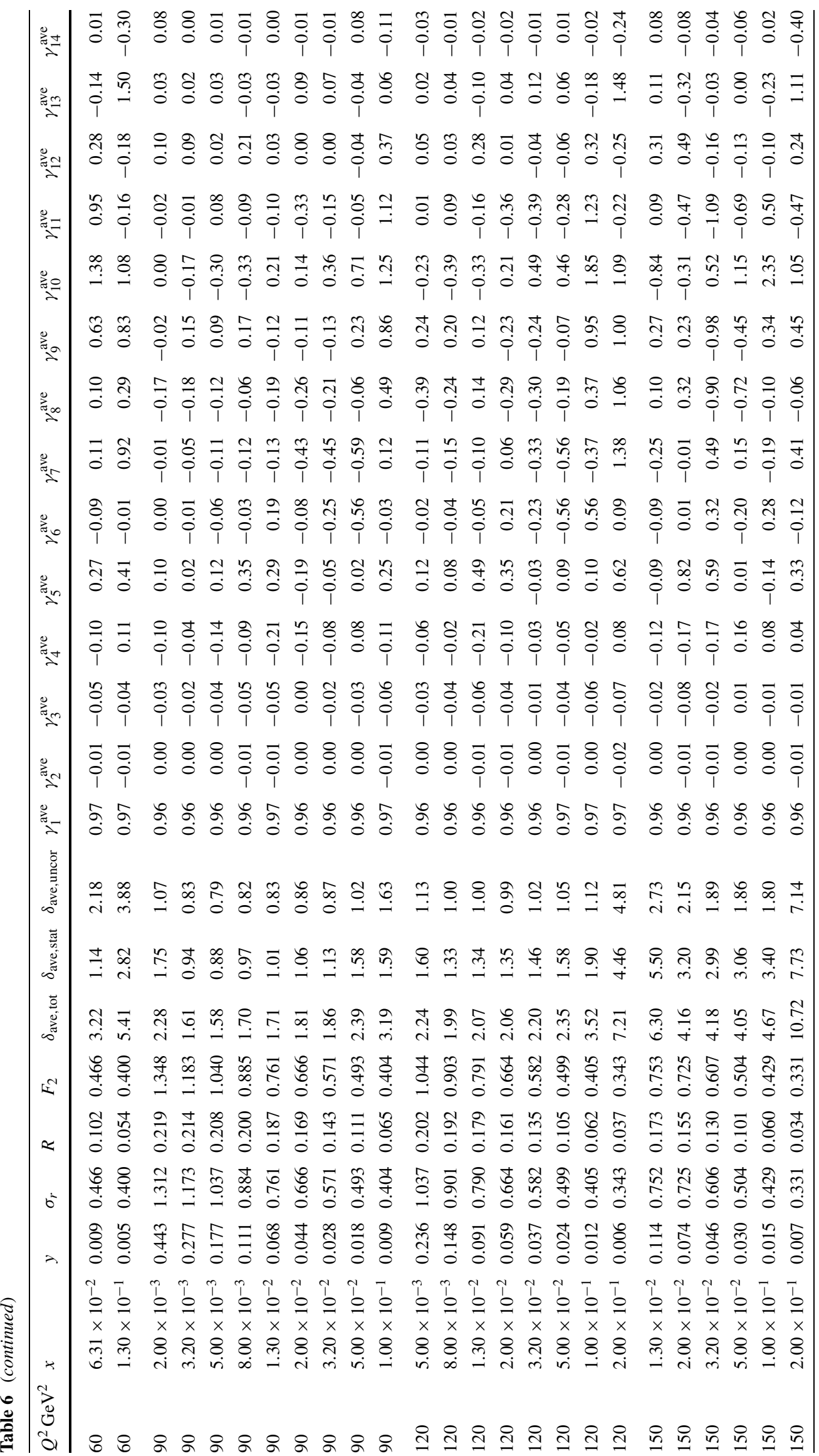


H1 Collaboration

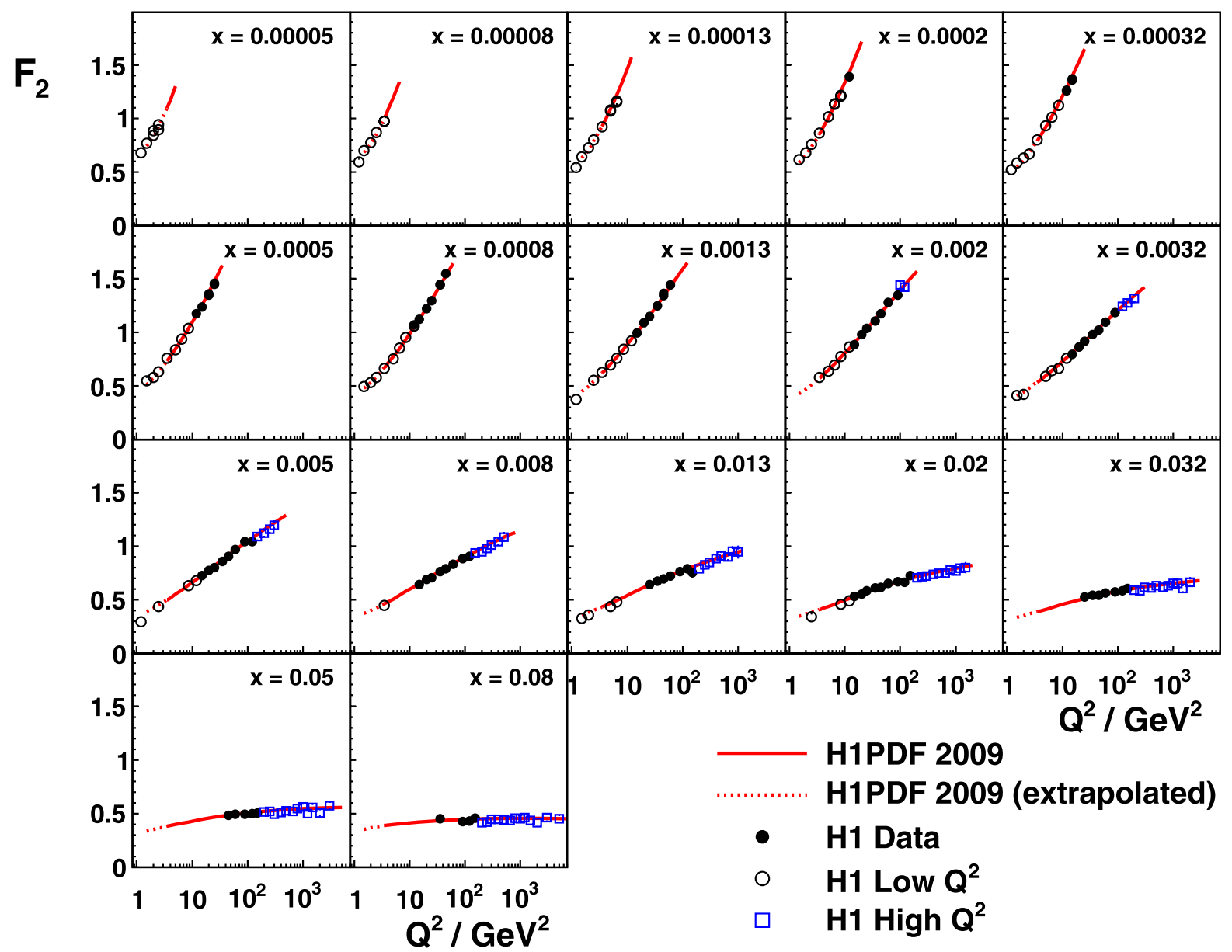

Fig. 6 Measurement of the structure function $F_{2}$ as a function of $Q^{2}$ at various values of $x$. The data of this measurement (closed circles) are complemented by the previously published data at low $Q^{2}$ (open circles) [2] and high $Q^{2}$ (open boxes) [9]. The error bars represent the total measurement uncertainties. The solid curve represents the QCD fit described in this paper for $Q^{2} \geq 3.5 \mathrm{GeV}^{2}$, which is also shown extrapolated down to $Q^{2}=1.5 \mathrm{GeV}^{2}$ (dashed)

The values used for $R=F_{L} /\left(F_{2}-F_{L}\right)$ are quoted in the cross section tables and are taken from the NLO QCD fit introduced below. Figure 5 shows the structure function $F_{2}$ at fixed $Q^{2}$ as a function of $x$ together with $\mathrm{H} 1$ data from lower [2] and higher $Q^{2}$ [9]. The data are well described by the NLO QCD fit. The rise of $F_{2}$ towards low $x$ is thus established at a much improved accuracy compared with the first observations $[28,29]$. There is no indication for a saturation of this behaviour in the $Q^{2}, x$ region of study.

Figure 6 shows the measurement of the structure function $F_{2}$ at fixed values of $x$ as a function of $Q^{2}$, compared with the QCD fit described below. The strong rise with $Q^{2}$ at low $x$ is a consequence of the large gluon density in this region. The data are well described by the QCD fit for $Q^{2} \geq 3.5 \mathrm{GeV}^{2}$. Also the extrapolation of the fit down to $Q^{2}=1.5 \mathrm{GeV}^{2}$ gives a reasonable description of the data. At the largest $x$ value covered by the data presented here, $x \simeq 0.1$, the structure function becomes almost independent of $Q^{2}$ as the result of a compensation of quark and gluon contributions to the $\ln Q^{2}$ derivative of $F_{2}$.

The DGLAP evolution equations [30-34] determine the derivative $\left(\partial F_{2} / \partial \ln Q^{2}\right)_{x}$ taken at fixed $x$, with the dominant low $x$ contribution arising from gluon splitting into a quark-anti-quark pair. The measurement of this derivative is a powerful constraint on the gluon distribution $x g$ and the strong coupling constant $\alpha_{s}$ [35]. A study of $\left(\partial F_{2} / \partial \ln Q^{2}\right)_{x}$ at low $x$ has been presented previously by the H1 Collaboration [1]. The method described there is used to determine this derivative for the new, combined $F_{2}$ data set, including the low $Q^{2}$ data [2]. The results are shown in Fig. 7 for different $x$ values as a function of $Q^{2}$. The dependence of the derivative on $Q^{2}$ is well reproduced by the QCD fit.

The rise of the structure function $F_{2}\left(x, Q^{2}\right)$ towards low $x$ may be quantified by the derivative $\lambda=-\left(\partial \ln F_{2} / \partial \ln x\right)_{Q^{2}}$ which is shown in Fig. 8. The result is more accurate than the previous measurement [6] and extends to lower $Q^{2}$. Within 
H1 Collaboration

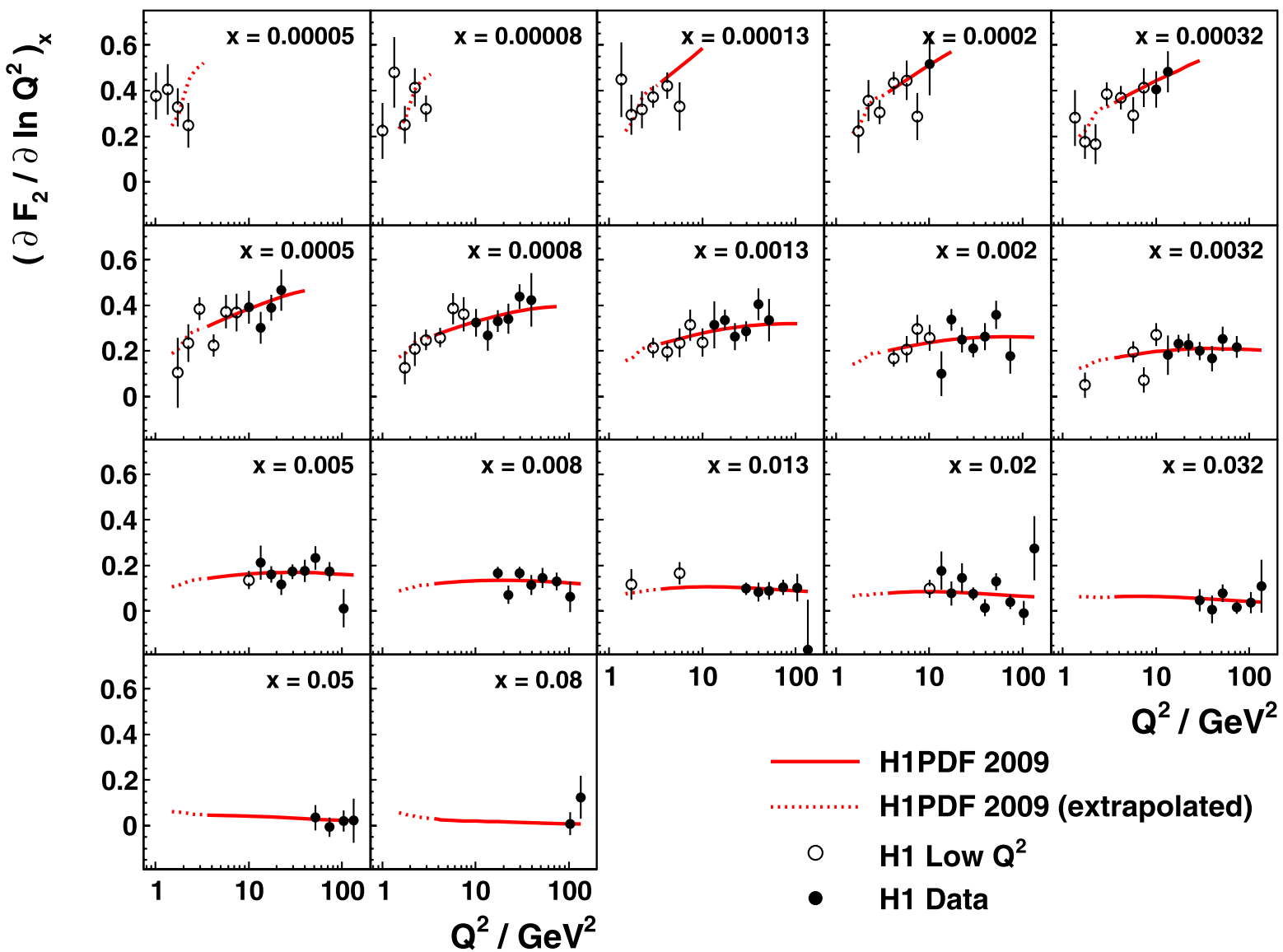

Fig. 7 Logarithmic $Q^{2}$ derivative of the structure function $F_{2}$ as a function of $Q^{2}$ at various values of $x$. The data of this measurement (closed circles) are complemented with the published data at lower $Q^{2}$ (open circles) [2]. The error bars represent the total measurement uncertainties. The solid curve represents the prediction of the QCD fit for $Q^{2} \geq 3.5 \mathrm{GeV}^{2}$, which is also shown extrapolated down to $Q^{2}=1.5 \mathrm{GeV}$ (dashed)

the uncertainty of the data, the derivative is constant at small $x<0.01$, i.e. $F_{2}$ for fixed $Q^{2}$ is consistent with a power law $F_{2} \propto x^{-\lambda}$. Small departures from this behaviour, as are inherent in the QCD fit, cannot be excluded either. The value of $\lambda$ increases from about 0.1 to 0.3 in the $Q^{2}$ region covered, from about 1 to $100 \mathrm{GeV}^{2}$. Data from [2] allow this measurement to be extended to $Q^{2}$ values below the region of validity of the DGLAP evolution.

\section{QCD analysis}

The neutral current cross section measurements presented here, together with the measurements at lower $Q^{2}$ [2] and the previously published $\mathrm{NC}$ and $\mathrm{CC}$ data at higher $Q^{2}$ [7-9], provide an accurate $\mathrm{H} 1$ data set for the determination of the parton density functions (PDFs) of the proton. A new QCD analysis, referred to as H1PDF 2009, is performed, which supersedes the previous H1PDF 2000 fit [9], as it relies on the more accurate new data. It also uses a general variable flavour number scheme (VFNS) treatment [36] of the heavy quarks, unlike the former fit, which used a zero mass variable flavour number scheme ("massless" scheme).

\subsection{Framework and settings}

The QCD analysis decomposes the structure functions into a set of parton densities: the gluon $x g$, the valence quarks, $x u_{v}$ and $x d_{v}$, and the combined anti-up type and anti-down type quarks, $x \bar{U}=x \bar{u}+x \bar{c}$ and $x \bar{D}=x \bar{d}+x \bar{s}+x \bar{b}$. These are parameterised ${ }^{2}$ at a starting scale $Q_{0}^{2}$ and are evolved using the DGLAP evolution equations. An adjustment of the parton distribution parameters is performed to best fit the measured cross sections.

\footnotetext{
${ }^{2}$ The previous H1PDF 2000 fit used a very similar decomposition of the quark flavours, but instead of the valence quark distributions $x u_{v}$ and $x d_{v}$, the combined up and down quark distributions $x U$ and $x D$ were used. These determine $x u_{v}=x U-x \bar{U}$ and $x d_{v}=x D-x \bar{D}$ assuming symmetry between the sea-quarks and anti-quarks for each flavour.
} 
Fig. 8 Measurement of the function $\lambda\left(x, Q^{2}\right)$, defined as the negative logarithmic $x$ derivative of $\ln F_{2}$ as a function of $x$ at various values of $Q^{2}$. The data of this measurement (closed circles) are complemented with the published data at lower $Q^{2}$ (open circles) [2]. The error bars represent the total measurement uncertainties. The solid curve represents the prediction of the QCD fit for $Q^{2} \geq 3.5 \mathrm{GeV}^{2}$, which is also shown extrapolated down to $Q^{2}=1.5 \mathrm{GeV}^{2}$ (dashed)

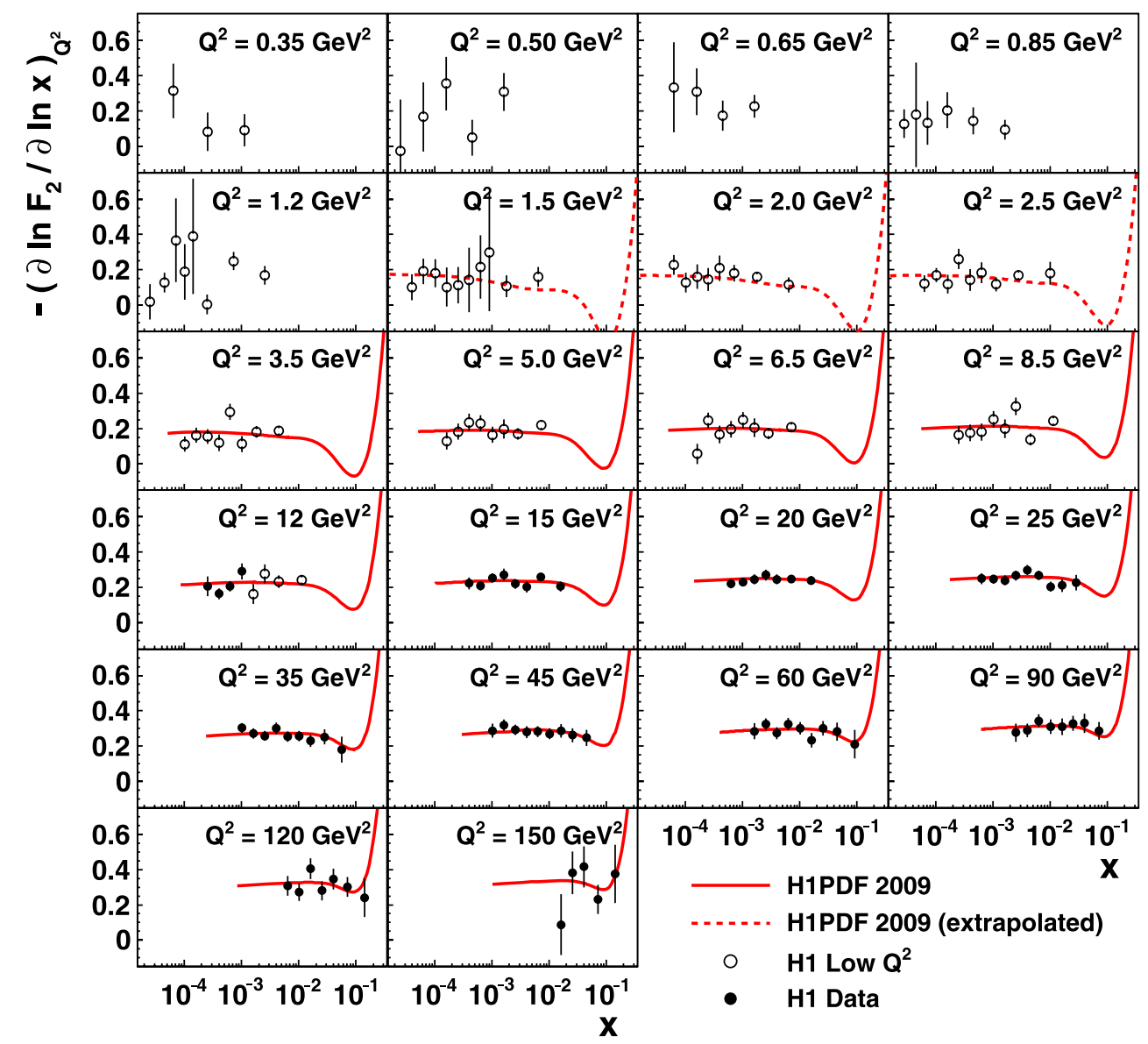

The analysis is performed at NLO within the $\overline{M S}$ renormalisation scheme. The program QCDNUM [37] is used to solve the evolution equations. From the evolved parton distributions, the structure functions are calculated in the VFNS scheme [36, 38], using $O\left(\alpha_{s}^{2}\right)$ coefficient functions for the calculation of $F_{L}$. The factorisation and renormalisation scales are both set to $Q^{2}$. The $\chi^{2}$ function is defined as for the cross section averaging discussed in Sect. 5.2 and [2]. It is minimised using the MINUIT package. The correlations between data points caused by systematic uncertainties are taken into account, following the numerical method presented in $[39,40]$. The correlations between the systematic error sources of the different high $Q^{2}$ data sets are treated as described in Table 2 in [9]. The error sources of the data presented here and of the lower $Q^{2}$ data [2] are all taken to be uncorrelated with any other source. The only exception is a $0.5 \%$ luminosity uncertainty, which is associated with the theoretical calculation of the Bethe-Heitler cross section and which is common to all $\mathrm{H} 1$ cross section measurements.

Following [41], the masses of the charm and beauty quarks are set to $m_{c}=1.4 \mathrm{GeV}$ and $m_{b}=4.75 \mathrm{GeV}$. The value of the strong coupling constant is taken to be $\alpha_{S}\left(M_{Z}^{2}\right)=0.1176$ [42]. The starting scale $Q_{0}^{2}$ is chosen to be slightly below the charm threshold, $Q_{0}^{2}=1.9 \mathrm{GeV}^{2}$. Hence at the starting scale the anti-quark densities simplify to $x \bar{U}(x)=x \bar{u}(x)$ and $x \bar{D}(x)=x(\bar{d}(x)+\bar{s}(x))$. The antistrange quark density, $x \bar{s}(x)=f_{s} x \bar{D}(x)$, is taken to be a constant fraction, $f_{s}=0.31$, of $x \bar{D}$ at the starting scale [8]. A cut $Q^{2}>Q_{\min }^{2}=3.5 \mathrm{GeV}^{2}$ is applied in order to ensure that the data used in the fit correspond to a kinematic domain where leading twist perturbative QCD can be used to predict the cross sections. Variations around these central values are taken into account as model uncertainties, as described below.

\subsection{Parameterisation}

The parton distributions $x P$ are parameterised at $Q_{0}^{2}$ using the general form

$x P(x)=A_{P} x^{B_{P}}(1-x)^{C_{P}}\left[1+D_{P} x+E_{P} x^{2}+\cdots\right]$.

The specific choice of the parameterisations is obtained as follows: the parameters $D, E, \ldots$ are added one-by-one in an iterative procedure and kept only, if they reduce the $\chi^{2}$ by more than 3 units. Parameterisations obtained are discarded 
Table 7 For each data set used in the H1PDF 2009 fit, the number of data points is shown, along with the $\chi^{2}$ contribution determined using the uncorrelated errors only $\left(\chi_{\text {unc }}^{2}\right)$

\begin{tabular}{llcr}
\hline Data set & & Data points & $\chi_{\text {unc }}^{2}$ \\
\hline Low $Q^{2}$ & {$[2]$} & 58 & 55.9 \\
Medium $Q^{2}$ & This measurement & 99 & 81.5 \\
$e^{+} p$ NC high $Q^{2}, 94-97$ & {$[7]$} & 130 & 92.6 \\
$e^{+} p$ CC high $Q^{2}, 94-97$ & {$[7]$} & 25 & 21.2 \\
$e^{-} p$ NC high $Q^{2}, 98-99$ & {$[8]$} & 139 & 112.1 \\
$e^{-} p$ CC high $Q^{2}, 98-99$ & {$[8]$} & 28 & 17.3 \\
$e^{+} p$ NC high $Q^{2}, 99-00$ & {$[9]$} & 147 & 137.4 \\
$e^{+} p$ CC high $Q^{2}, 99-00$ & {$[9]$} & 28 & 31.1 \\
\hline
\end{tabular}

if the structure functions $F_{2}$ and $F_{L}$ are negative anywhere in the range $10^{-5}<x<1$ for $Q^{2}>Q_{0}^{2}$. Parameterisations leading to very low valence quark distributions as compared to the total sea-quark density $x S(x)=2 x(\bar{U}(x)+\bar{D}(x))$ at large $x$, which dramatically fail to describe $v p$ and $v d$ fixed target measurements of the structure function $x F_{3}$ [43], are also not considered. Some parameterisations of the $x u_{v}$ density involving a quadratic $E$ or cubic $F$ term in $x$ in the expansion (5) are examples of these. They are, however, included in the parameterisation uncertainty discussed below. The same applies to fits where the parton distributions are negative at very high $x$, as happens for some parameterisations of the gluon density involving an $E$ or $F$ term. Among the possible parameterisations the one with the lowest $\chi^{2}$ is selected. This procedure, at $Q_{0}^{2}=1.9 \mathrm{GeV}^{2}$, leads to the following choice:

$$
\begin{aligned}
& x g(x)=A_{g} x^{B_{g}}(1-x)^{C_{g}}\left[1+D_{g} x\right], \\
& x u_{v}(x)=A_{u_{v}} x^{B_{u_{v}}}(1-x)^{C_{u_{v}}}, \\
& x d_{v}(x)=A_{d_{v}} x^{B_{d_{v}}}(1-x)^{C_{d_{v}}} \\
& x \bar{U}(x)=A_{\bar{U}} x^{B_{\bar{U}}}(1-x)^{C_{\bar{U}}} \\
& x \bar{D}(x)=A_{\bar{D}} x^{B_{\bar{D}}}(1-x)^{C_{\bar{D}}}
\end{aligned}
$$

The normalisation parameters $A_{u_{v}}$ and $A_{d_{v}}$ are not fitted, but are obtained from the other parameters via the quark counting rules. Since the existing data have a limited sensitivity to the behaviour of the valence quark distributions at low $x$, it is assumed that $B_{u_{v}}=B_{d_{v}}$. Similarly, the behaviour of the up and down anti-quarks at low $x$ is assumed to be governed by the same power, $B_{\bar{U}}=B_{\bar{D}}$. As in [9], the normalisations of the $\bar{U}$ and $\bar{D}$ distributions are related by $A_{\bar{U}}=A_{\bar{D}}\left(1-f_{s}\right)$ which corresponds to the usual assumption that $\bar{d} / \bar{u} \rightarrow 1$ as $x \rightarrow 0$. Finally, the normalisation $A_{g}$ of the gluon distribution is derived from the momentum sum rule. The total number of free parameters is thus equal to ten.
Table 8 Fitted parameters corresponding to the distributions $x g(x)$, $x u_{v}(x), x d_{v}(x), x \bar{U}(x)$ and $x \bar{D}(x)$ at the starting scale $Q_{0}^{2}=1.9 \mathrm{GeV}^{2}$ (see Sect. 6.2). The symbol * indicates that the corresponding parameter is not a free parameter of the fit, but is derived from the other parameters

\begin{tabular}{lllrl}
\hline$x P$ & $A_{P}$ & $B_{P}$ & \multicolumn{1}{c}{$C_{P}$} & $D_{P}$ \\
\hline$x g$ & $5.66^{*}$ & 0.243 & 18.76 & 34.0 \\
$x u_{v}$ & $5.15^{*}$ & 0.784 & 3.25 & - \\
$x d_{v}$ & $3.29^{*}$ & $0.784^{*}$ & 4.77 & - \\
$x \bar{U}$ & $0.105^{*}$ & -0.177 & 2.42 & - \\
$x \bar{D}$ & 0.152 & $-0.177^{*}$ & 3.42 & - \\
\hline
\end{tabular}

\subsection{Fit results}

The central fit of this analysis as specified above has a $\chi^{2}$ of 587 for 644 degrees of freedom. The $\chi^{2}$ value for each data set is given in Table 7 . No significant tension is observed between the fit results and the systematic uncertainties on the low, medium, and high $Q^{2}$ data sets.

The fitted parameters of the distributions at the starting scale are given in Table 8. The resulting parton distributions, including the total sea-quark density, are shown at $Q^{2}=4 \mathrm{GeV}^{2}$ in Fig. 9. The inner error band describes the experimental uncertainty, obtained from the criterion $\Delta \chi^{2}=1$ using the Hessian method as described in [40] and the numerical algorithm presented in [44]. The middle error band represents the experimental and model uncertainties added in quadrature. The model uncertainties are obtained by varying:

- the charm mass $m_{c}$ between $1.38 \mathrm{GeV}$ and $1.47 \mathrm{GeV}$;

- the bottom mass $m_{b}$ between $4.3 \mathrm{GeV}$ and $5.0 \mathrm{GeV}$;

- the strange fraction $f_{s}$ from 0.25 to 0.40 ;

- the value of $Q_{\min }^{2}$, from $2.25 \mathrm{GeV}^{2}$ to $5.0 \mathrm{GeV}^{2}$;

- the starting scale $Q_{0}^{2}$ down to $1.5 \mathrm{GeV}^{2}$. 
Fig. 9 Parton distributions as determined by the H1PDF 2009 QCD fit at $Q^{2}=4 \mathrm{GeV}^{2}$.

Shown are the combined up and down quark distributions,

$x U=x(u+c)$ and

$x D=x(d+s)$, their anti-quark counter parts, $x \bar{U}$ and $x \bar{D}$, the valence quark distributions, $x u_{v}$ and $x d_{v}$, the total sea distribution, $x S=2 x(\bar{U}+\bar{D})$, and the gluon distribution, $x g$.

The inner error bands show the experimental uncertainty, the middle error bands include the theoretical model uncertainties of the fit assumptions, and the outer error band represents the total uncertainty including the parameterisation uncertainty

Fig. 10 Parton distributions as determined by the H1PDF 2009 QCD fit at $Q^{2}=1.9 \mathrm{GeV}^{2}$ (a)-(b) and at $Q^{2}=10 \mathrm{GeV}^{2}$ (c)-(d). In (a) and (c) (linear vertical scale), the gluon and sea-quark densities are downscaled by a factor 0.05 . The inner error bands show the experimental uncertainty, the middle error bands include the theoretical model uncertainties of the fit assumptions, and the outer error band represents the total uncertainty including the parameterisation uncertainty, see text
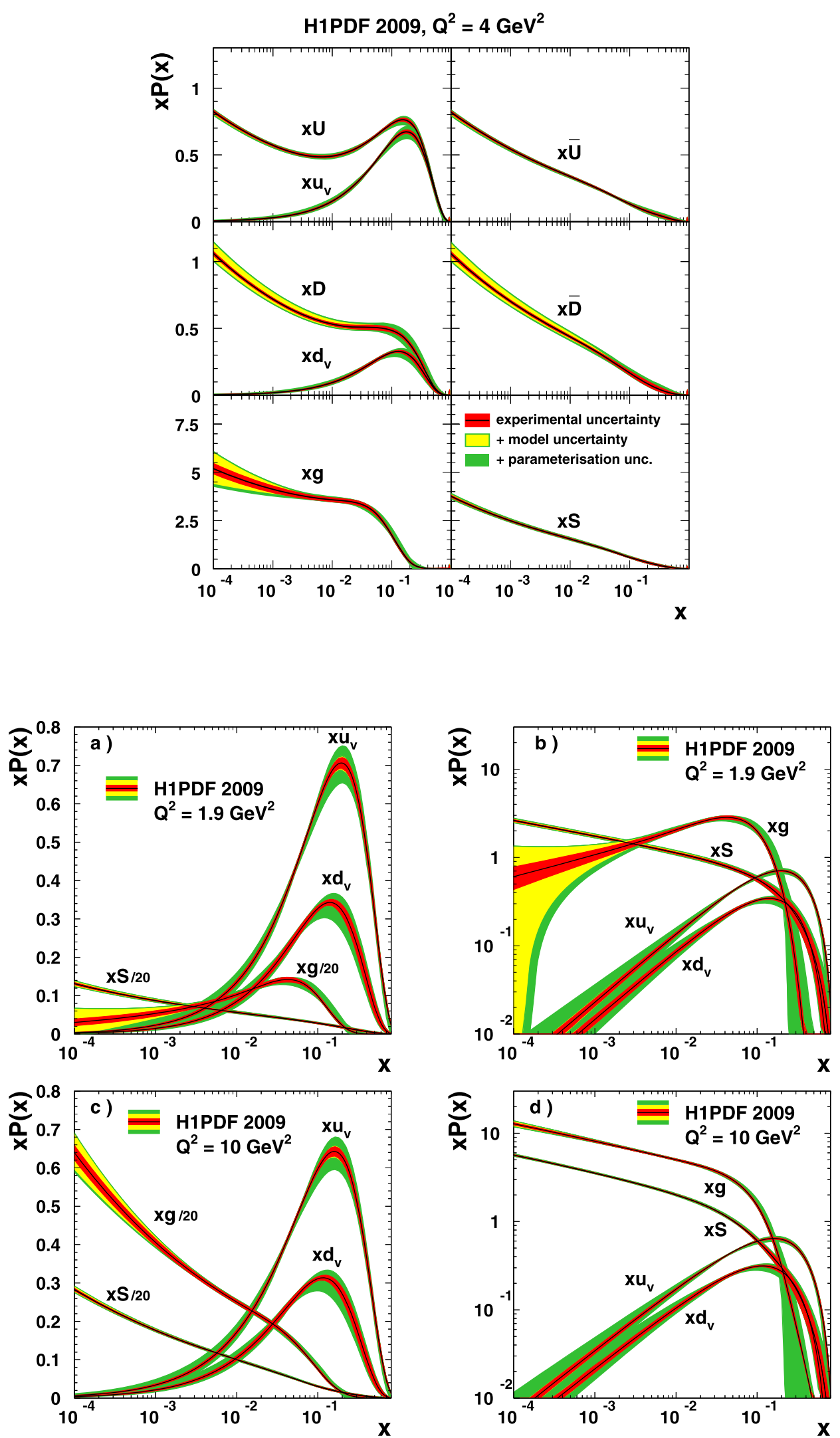
The resulting model uncertainty at low $x$ is dominated by the sensitivity of the fit to the $Q_{0}^{2}$ variation. ${ }^{3}$

In addition to the model uncertainty a new error contribution is introduced resulting from the parameterisation choice. As explained in Sect. 6.2, alternative parameterisations leading to good fit quality but peculiar behaviour at large $x$ are used to estimate the parametrisation uncertainties. An envelope of these fit solutions is built which is added in quadrature to the contributions of the experimental and model uncertainties.

The distributions of $x u_{v}(x), x d_{v}(x), x g(x)$ and $x S(x)$ are shown at the starting scale $Q^{2}=1.9 \mathrm{GeV}^{2}$ and at $Q^{2}=$ $10 \mathrm{GeV}^{2}$ in Figs. 10(a)-(b) and Figs. 10(c)-(d), respectively, with both linear and logarithmic scales. A comparison of the PDFs at the starting scale with their behaviour in the DIS region, here represented by $Q^{2}=10 \mathrm{GeV}^{2}$, illustrates the rather dramatic influence of the DGLAP evolution on the sea-quark and gluon densities. At $Q^{2}=1.9 \mathrm{GeV}^{2}$ the seaquark density rises towards low $x$, in contrast to the gluon distribution which has a valence quark-like shape. The $Q^{2}$ evolution rapidly changes the low- $x$ behaviour of the gluon distribution, which starts to rise similarly to the sea-quark distribution towards low $x$. In contrast, the non-singlet valence quark distributions evolve very slowly, as expected. As is shown in Figs. 10 (c)-(d), $x g$ is the dominating parton distribution at low $x$ and higher $Q^{2}$, here displayed for $Q^{2}=10 \mathrm{GeV}^{2}$.

\section{Summary}

A new measurement is presented of the inclusive double differential cross section for deep inelastic positron-proton scattering, $e^{+} p \rightarrow e^{+} X$, in the region of small Bjorken $x$, $2 \times 10^{-4} \leq x \leq 0.1$, and four-momentum transfer squared, $12 \mathrm{GeV}^{2} \leq Q^{2} \leq 150 \mathrm{GeV}^{2}$. The data, corresponding to an integrated luminosity of about $22 \mathrm{pb}^{-1}$, were obtained with the $\mathrm{H} 1$ detector at the $e p$ collider HERA at beam energies $E_{e}=27.6 \mathrm{GeV}$ and $E_{p}=920 \mathrm{GeV}$. A small bias in a similar previously published data set, taken at $E_{p}=820 \mathrm{GeV}$, is found and corrected. The two data sets are then combined and represent the most precise measurement in this kinematic region to date, with typical total uncertainties in the range of $1.3-2 \%$.

The kinematic range of the measurement corresponds to a wide range of inelasticity $y$, from 0.005 to 0.6 . The data are

\footnotetext{
${ }^{3}$ An uncertainty contribution due to the $Q_{0}^{2}$ variation is calculated by allowing $Q_{0}^{2}$ to be as low as $1.5 \mathrm{GeV}^{2}$. This causes an increase of the $\chi^{2}$, which is comparable to the $\chi^{2}$ change in a massless fit, when $Q_{0}^{2}$ is varied from $4 \mathrm{GeV}^{2}$ to $2 \mathrm{GeV}^{2}$, as has conventionally been done in the past. Since the VFNS scheme implementation requires $Q_{0}^{2} \leq m_{c}^{2}$, a default of $Q_{0}^{2}=1.9 \mathrm{GeV}^{2}$ is chosen and the calculated uncertainty is symmetrised.
}

used to determine the structure function $F_{2}\left(x, Q^{2}\right)$, which is observed to rise continuously towards low $x$ at fixed $Q^{2}$.

The high precision of the data allows new measurements to be presented of the partial derivatives of $F_{2}\left(x, Q^{2}\right)$ with respect to $Q^{2}$ and $x$. For $x<0.01$, the derivative of $\ln F_{2}$ with respect to $\ln x$ confirms a power law dependence of $F_{2} \propto x^{-\lambda}$ with $\lambda$ depending primarily on $Q^{2}$. The derivative of $F_{2}$ with respect to $\ln Q^{2}$ is a measure for the gluon distribution $x g$ at low $x$.

An NLO QCD fit to the H1 data alone, including the new measurements presented in this paper, is performed. The fit implements a variable flavour treatment of heavy quark threshold effects. This new H1PDF 2009 fit supersedes the H1PDF 2000 previously obtained and provides a new determination of the gluon and quark densities of the proton including experimental, model, and parameterisation uncertainties.

Acknowledgements We are grateful to the HERA machine group whose outstanding efforts have made this experiment possible. We thank the engineers and technicians for their work in constructing and maintaining the $\mathrm{H} 1$ detector, our funding agencies for financial support, the DESY technical staff for continual assistance and the DESY directorate for support and for the hospitality which they extend to the non DESY members of the collaboration. We thank R. Thorne for useful discussions and for providing us with the code to calculate the structure functions in the variable flavour number scheme.

\section{References}

1. C. Adloff et al. (H1 Collaboration), Eur. Phys. J. C 21, 33 (2001). hep-ex/0012053

2. F.D. Aaron et al. (H1 Collaboration), Eur. Phys. J. C 63, 625 (2009)

3. S. Chekanov et al. (ZEUS Collaboration), Eur. Phys. J. C 21, 443 (2001). hep-ex/0105090

4. S. Chekanov et al. (ZEUS Collaboration), Phys. Lett. B (2009, submitted). arXiv:0904.1092

5. F.D. Aaron et al. (H1 Collaboration), Phys. Lett. B 665, 139 (2008). arXiv:0805.2809 [hep-ex]

6. C. Adloff et al. (H1 Collaboration), Phys. Lett. B 520, 183 (2001). hep-ex/0108035

7. C. Adloff et al. (H1 Collaboration), Eur. Phys. J. C 13, 609 (2000). hep-ex/9908059

8. C. Adloff et al. (H1 Collaboration), Eur. Phys. J. C 19, 269 (2001). hep-ex/0012052

9. C. Adloff et al. (H1 Collaboration), Eur. Phys. J. C 30, 1 (2003). hep-ex/0304003

10. J. Kretzschmar, Doctoral thesis, Humboldt-Universität zu Berlin (2008). http://edoc.hu-berlin.de/dissertationen/kretzschmar-jan2008-02-04

11. I. Abt et al. (H1 Collaboration), Nucl. Instrum. Methods A 386, 310 (1997)

12. I. Abt et al. (H1 Collaboration), Nucl. Instrum. Methods A 386, 348 (1997)

13. R.D. Appuhn et al. (H1 SpaCal Group), Nucl. Instrum. Methods A 386, 397 (1997)

14. B. Andrieu et al. (H1 Calorimeter Group), Nucl. Instrum. Methods A 336, 460 (1993)

15. K. Müller et al., Nucl. Instrum. Methods A 312, 457 (1992) 
16. S. Eichenberger et al., Nucl. Instrum. Methods A 323, 532 (1992)

17. D. Pitzl et al., Nucl. Instrum. Methods A 454, 334 (2000). hep-ex/0002044

18. W. Eick et al., Nucl. Instrum. Methods A 386, 81 (1997)

19. B. Andrieu et al. (H1 Calorimeter Group), Nucl. Instrum. Methods A 336, 499 (1993)

20. T.C. Awes et al., Nucl. Instrum. Methods A 311, 130 (1992)

21. G.A. Schuler, H. Spiesberger, in Physics at HERA, vol. 3, ed. by W. Buchmüller, G. Ingelman (1991), pp. 1419-1432, DJANGOH 1.4

22. R. Engel, J. Ranft, Phys. Rev. D 54, 4244 (1996), PHOJET 1.0. hep-ph/9509373

23. A. Courau, P. Kessler, Phys. Rev. D 46, 117 (1992), COMPTON 2.2

24. A. Kwiatkowski, H. Spiesberger, H.-J. Möhring, Comput. Phys. Commun. 69, 155 (1992)

25. A. Arbuzov et al., Comput. Phys. Commun. 94, 128 (1996)

26. R. Brun et al., GEANT3 User's Guide, CERN (1987). CERN$\mathrm{DD} / \mathrm{EE}$ 84-1

27. S. Glazov, in 13th International Workshop on Deep Inelastic Scattering; DIS 2005, ed. by W. Smith, S.R. Dasu, AIP Conference Proceedings (2005)
28. I. Abt et al. (H1 Collaboration), Nucl. Phys. B 407, 515 (1993)

29. M. Derrick et al. (ZEUS Collaboration), Phys. Lett. B 316, 412 (1993)

30. V.N. Gribov, L.N. Lipatov, Sov. J. Nucl. Phys. 15, 438 (1972)

31. V.N. Gribov, L.N. Lipatov, Sov. J. Nucl. Phys. 15, 675 (1972)

32. L.N. Lipatov, Sov. J. Nucl. Phys. 20, 94 (1975)

33. Y.L. Dokshitzer, Sov. Phys. JETP 46, 641 (1977)

34. G. Altarelli, G. Parisi, Nucl. Phys. B 126, 298 (1977)

35. P.W. Johnson, W. Tung, Phys. Rev. D 16, 2769 (1977)

36. R.S. Thorne, Phys. Rev. D 73, 054019 (2006). hep-ph/0601245

37. M. Botje, QCDNUM version $17 \beta$, available at http://www. nikhef.nl/ h24/qcdnum/index.html

38. R. Thorne, Fortran code, private communication

39. C. Pascaud, F. Zomer, Preprint LAL-95-05

40. J. Pumplin et al., J. High Energy Phys. 07, 012 (2002). hep-ph/ 0201195

41. A.D. Martin, W.J. Stirling, R.S. Thorne, G. Watt (2009). arXiv:0901.0002 [hep-ph]

42. C. Amsler et al. (Particle Data Group), Phys. Lett. B 667, 1 (2008)

43. D. Allasia et al., Z. Phys. C 28, 321 (1985)

44. J. Pumplin, D.R. Stump, W. Tung, Phys. Rev. D 65, 014011 (2002). hep-ph/0008191 